\title{
Survey on the Inverse Spectral Problem*
}

\section{by Steve Zelditch ${ }^{\dagger}$}

Let $(M, g)$ be a Riemannian manifold of dimension $n$, possibly with boundary, and denote its Laplacian by

$$
\Delta_{g}=\frac{1}{\sqrt{g}} \sum_{i, j=1}^{n} \frac{\partial}{\partial x_{i}} g^{i j} \sqrt{g} \frac{\partial}{\partial x_{j}},
$$

where $g_{i j}=g\left(\frac{\partial}{\partial x_{i}}, \frac{\partial}{\partial x_{j}}\right),\left[g^{i j}\right]$ is the inverse matrix to $\left[g_{i j}\right]$ and $g=\operatorname{det}\left[g_{i j}\right]$. When $M$ is compact the spectrum of $\Delta_{g}$ is the discrete set of eigenvalues

$$
\left\{\begin{array}{l}
-\Delta_{g} \varphi_{j}=\lambda_{j}^{2} \varphi_{j},\left\langle\varphi_{i}, \varphi_{j}\right\rangle=\delta_{i j}, \\
B \varphi_{j}=0 \text { on } \partial M,
\end{array}\right.
$$

repeated according to their multiplicities. When $M$ has a non-empty boundary $\partial M$, one imposes boundary conditions such as Dirichlet $B u=\left.u\right|_{\partial M}$ or Neumann $B u=\left.\partial_{\nu} u\right|_{\partial M}$.

More generally, we may consider the eigenvalue problem for semi-classical Schrödinger operators

$$
\hat{H}_{\hbar} \psi_{\hbar, j}:=\left(-\frac{\hbar^{2}}{2} \Delta_{g}+V\right) \psi_{\hbar, j}=E_{j}(\hbar) \psi_{\hbar, j},
$$

where $V \in C^{\infty}(M)$ is a potential. Here, $\hbar$ is the Planck constant. When $(M, g)$ is non-compact, the spectrum of $\Delta_{g}$ is often continuous, and one might instead pose the inverse problem for eigenvalues of the scattering operator $S_{\hbar}$ ("phase shifts") or poles of its analytic continuation ("resonances") $§ 6$. If the potential $V$ grows at infinity, $\hat{H}_{\hbar}$ has a discrete spectrum.

The inverse spectral or scattering problem is to determine as much as possible of the metric, potential or domain from spectral or scattering data. In the

\footnotetext{
* Research partially supported by NSF grant DMS-1206527.

† Department of Mathematics, Northwestern University, Evanston, Illinois, U.S.A.

E-mail: zelditch@math.northwestern.edu
}

case of discrete spectrum one would like to "invert" the map $S p:(M, g, V) \rightarrow \operatorname{Spec}\left(\hat{H}_{\hbar}\right)=\left\{\lambda_{j}^{2}\right\}_{j=1}^{\infty}$, or at least to show that it is 1-1 when restricted to a reasonable class of $(M, g, V)$. We note that there are two types of inverse problems for Schrödinger operators (2): (i) the more difficult one where $\hbar$ is fixed, and (ii) the substantially simpler one where one attempts to determine the potential from the 1-parameter family of spectra $\left\{E_{j}(\hbar)\right\}_{j=1}^{\infty}$. In some sense, one would like to use the $\lambda_{j}$ as "coordinates" on the "space" of isometry classes of metrics, potentials or domains. This is only a heuristic way to envision the problem because of its infinite dimensionality. Except in dimension one, the range of $S p$ is not known and is certainly a very small subset of the space $\Sigma \subset \mathbb{R}_{+}^{\mathbb{N}}$ of sequences compatible with the Weyl law for counting eigenvalues, which in the purely metric case takes the form $\#\left\{j: \lambda_{j} \leq \lambda\right] \sim C_{n} \operatorname{Vol}(M, g) \lambda^{n}$. However it leads one to ask if $S p$ is "generically" $1-1$, to study its first and second derivative, to describe its level sets (isospectral sets), or to see if there is any natural additional data one may use to supplement $S p$ to make it a wellbehaved map. A simpler problem is spectral rigidity: can one deform a metric, potential or domain in a non-trivial way while keeping the eigenvalues fixed? I.e. do there exist (smooth) curves in an isospectral set of domains, metrics or potentials with a fixed spectrum? Isometric domains or metrics are regarded as the same, or as trivially isospectral, and when we discuss isospectral domains or metrics it is understood that they are non-trivially isospectral.

\subsection{Historical Background}

The inverse spectral problem was first stated by Sir Arthur Schuster in 1882 (see Appendix F of [St] 
(p. 395)): “...Find out the shape of the bell by means of the sound which it is capable of sending out. And this is the problem which ultimately spectroscopy hopes to solve in the case of light. In the meantime we must welcome with delight even the smallest step in the right direction." An early result is that of Rayleigh, determining the coefficients of a differential operator from its eigenvalues [R]. The problem is best known in the formulation of L. Bers, S. Bochner, and M. Kac ("Can you hear the shape of a drum"). Here, the metric is assumed to be Euclidean, $V=0$ and one wants to determine a domain $\Omega \subset \mathbb{R}^{2}$ from its Dirichlet or Neumann eigenvalues.

In quantum physics, the inverse problem is mainly to determine the potential (or metric) of (2) from it spectral and scattering data [N, N2, ChS]. The idea is that this data is observable in experiments while the potential is not. There are many types of scattering data but in this survey we only discuss the phase shifts or eigenvalues of the scattering matrix $S_{h}$ (a unitary operator acting on the sphere; $\S 6)$. The problem of recovering the potential of a Schrödinger operator from eigenvalues and phase shifts was studied since the 1940's when V. Bargmann discovered two potentials with the same phase shifts [Bar1, Bar2]. The inverse problem in dimension one was solved with some restrictions by Gel'fand-Levitan and Marchenko (see [GL, Mar, Mar2] and also [Dy, Dy2] for background and history). Some other early articles are $[\mathrm{A}, \mathrm{Bo}, \mathrm{Hy}, \mathrm{L}]$.

The inverse spectral problem in one dimension is simpler than and quite different from the problem in higher dimensions and in this survey we only consider the problem when $\operatorname{dim} M \geq 2$. To give an idea of how the difficulty grows with the dimension, it is simple to prove that the standard metric $g_{0}$ on the sphere $S^{n}$ is determined by the eigenvalues of $\Delta_{g_{0}}$ when $n=2$. It is difficult but possible to show $g_{0}$ is determined by $\operatorname{Spec}\left(\Delta_{g_{0}}\right)$ for $n \leq 6$ (Tanno). It is unknown whether $g_{0}$ is determined by $\operatorname{Spec}\left(\Delta_{g_{0}}\right)$ when $n \geq 7$, although they are known to be spectrally rigid and locally determined (Tanno). On the other hand, it is known that balls in $\mathbb{R}^{n}$ are determined by their eigenvalues (by the isoperimetric inequality).

There exist two broad classes of results in inverse spectral theory: (a) construction of isospectral pairs or deformations, i.e. of "counter-examples" to the inverse spectral problem; (b) positive results proving rigidity or spectral determination on classes of domains, metrics or potentials. In this survey we concentrate on positive results of type (b) and ask, how much of the geometry is determined by the spectrum? We refer to [GP] for some results of type (a).

There are a number of recent surveys on the inverse spectral problem [DH, Z1]. The present survey is shorter and less systematic. Its purpose is to give a reader who is not very familiar with inverse spectral theory an idea of the current state of the art as well as some idea of what is involved in proving inverse spectral results. Some are new and some are relatively old but illustrate different features of the problem. In particular, we do not attempt to provide a comprehensive list of references (see [DH, Z1]). One of the charms of the subject is that the problems have an obvious motivation in both mathematics and physics, are easy to state, yet remain largely open after 50 years. After reviewing the basic techniques we state some tantalizingly simple sounding problems in $§ 7$.

\subsection{Analyticity, Symmetry, Accidental Degeneracies and Multiple Spectra}

The inverse spectral problem in higher dimensions involves a vast collection of objects and it is not surprising that there are few general results applying to all of them. Inevitably there exists sporadic spectral behavior that allows isospectral sets to have surprising properties. For instance, the eigenvalues or lengths of closed geodesics might have unusual multiplicities. In the theory of automorphic forms, the Langlands (-Eichler) correspondence between arithmetic hyperbolic surfaces with cusps and compact arithmetic surfaces defined by quaternion algebras is a (partial) isospectrality phenomenon that has no known global geometric interpretation. The correspondence is proved by "matching orbital integrals", e.g. by comparing lengths of closed geodesics on both surfaces. Thus, the partial isospectrality has arithmetic rather than geometric origins.

It is common to add assumptions or restrictions in order to prove positive results. Before introducing definitions and results, we call the reader's attention to some common ones.

- (A) The potentials, metrics or domains are often assumed to have a symmetry, either discrete or continuous. On $\mathbb{R}^{n}$, potentials or domains are assumed to be invariant under reflections $x_{j} \rightarrow-x_{j}$, or under a di-hedral symmetry [Z2]. Continuous symmetry often takes the form of radial potentials, surfaces of revolution etc. The inverse spectral problem is restricted to the class of such symmetric domains or potentials.

- (B) The metrics, potentiasl or domains are often assumed to be real analytic. Convexity is another natural condition. In the counter-example of [GWW] of non-isospectral domains with the same Dirichlet (resp. Neumann) spectra, the domains have corners and are non-convex.

- (C) The isospectrality is often assumed to occur for more than one spectrum, as in the case of (2) for the one-parameter family $\hbar \rightarrow E_{j}(\hbar)$ of spectra. 
Or in the case of domains, one might prescribe both the Dirichlet and Neumann spectra, or both the spectrum "inside" the domain and also "outside" of it.

- (D) In counter-examples, the metric, potential or domain often has some non-generic behavior such as high-dimensional manifolds of closed geodesics or other mulitplicities in the length spectrum. Most positive results require multiplicity one in the length spectrum.

Let us explain the background to these assumptions.

In the early days of one-dimensional inverse spectral theory, Borg [Bo], Levinson [L] and Marchenko [Mar, Mar2] proved that a potential $V \in L^{1}[0, L]$ is determined by two spectra of a Schrödinger operator (somewhat similar to prescribing spectra of (2) for two values of $\hbar$ ). When the potential is symmetric, $q(L-x)=q(x)$ then one good spectrum, either the Dirichlet or Neumann spectrum, suffices (see e.g. $[\mathrm{BrH}])$. The solution is not unique for general nonsymmetric potentials. Results assuming $\mathbb{Z}_{2}$ symmetries are multi-dimensional generalizations of this result. See [Z2, GU, He, HZ2] for recent results of this kind.

Radial symmetry is a very common assumption in inverse scattering theory since it essentially reduces the inverse problem to one dimension [KKS, N3]. The author is not aware of any inverse results for phase shifts that do not assume radial symmetry. The analogous problem for metrics is to assume the surface has an $S^{1}$ rotational symmetry, i.e. is a surface of revolution. When it is also mirror symmetric, the inverse problem was solved in $[\mathrm{BrH}]$ by reducing the inverse problem to that of Borg-Levinson-Marchenko. There are several other results of this kind due to M. Kac, D. Stroock and P. Bérard (see [DH]). In [Z3] the author solved the inverse spectral problem for convex analytic surfaces of revolution without mirror symmetry; the proof used what we now call a normal forms approach (Theorem 3.4 and §4.4).

A plane domain may be locally defined as the graph of a function $y=f(x)$ over $(-1,1)$ and therefore has the same number of functional degrees of freedom as a surface of revolution or a radial potential. I.e. the unknown is a function of one real variable. If the domain has the $\mathbb{Z}_{2} \times \mathbb{Z}_{2}$ symmetries of an ellipse, the problem has the same symmetries as the one dimensional Schrödinger problem of Borg-Levinson. The lack of uniqueness in the $1 \mathrm{D}$ case might suggest lack of uniqueness for the plane domain problem without two symmetries. However, if the domains are real analytic, the one $\mathbb{Z}_{2}$ symmetry is enough to solve the inverse problem for the Dirichlet or Neumann spectrum (Theorem 3.1 [Z2]). It is not known at present if the inverse spectral problem is uniquely solvable (even in the analytic class) without assuming at least one symmetry.

The history of results for radial potentials or surfaces of revolution might also suggest that one can only solve the inverse problem if the unknown is a function of just one real variable, e.g. that one cannot "hear the shape of a multi-dimensional drum". However this is also not true. In [HZ] H. Hezari and the author proved that one can determine a real analytic $\mathbb{Z}_{2}^{n}$-symmetric domain in $\mathbb{R}^{n}$ for any $n$ from its Dirichlet or Neumann spectrum (Theorem 3.3); here the unknown is a function $f\left(x_{1}, \ldots, x_{n}\right)$ of $n$ variables. An earlier result for the $\hbar$ family of spectra of (2) for $\mathbb{Z}_{2}^{n}$-symmetric potentials was proved in [GU, He]; in [He] fewer symmetry assumptions were needed.

Real analyticity is often assumed because the spectral data often leads to local expressions in terms of Taylor coefficients of the metric, potential or domain around closed geodesics. Unless one is making a deformation, there is no obvious way to relate spectral information for distinct geodesics. Real analyticity is used to make a local result global, i.e. to use Taylor coefficients at one point to determine the unknown function.

The $\hbar$-problem is significantly simpler than the problem for a single spectrum. It is similar to assuming the knowledge of the spectrum of several commuting operators, e.g. the joint spectrum of $\Delta_{g}$ and of $L=\frac{\partial}{\partial \theta}$ on a surface of revolution; here $L$ generates rotations around the axis. In [Z2], the principal step is to show that the single spectrum of $\Delta_{g}$ determines the joint spectrum. This is clearly necessary if the inverse spectral problem for one value of $\hbar$ is solvable.

Regarding (D), we recall that isospectrality of two Laplacians $\Delta_{j}$ is the condition that there exists a unitary operator $U$ such that $U \Delta_{1} U^{*}=\Delta_{2}$. In the correspondence principle between classical and quantum mechanics, the classical condition is that there should exist a symplectic diffeomorphism $\chi$ between the cotangent bundles which restricts to a diffeomorphism $\chi: S_{g_{1}}^{*} M_{1} \rightarrow S_{g_{2}}^{*} M_{2}$ between the unit cosphere bundles and which conjugates the geodesic flows $G_{j}^{t}$ in the sense that $\chi G_{1}^{t} \chi^{-1}=G_{2}^{t}$. The correspondence principle only works if $U$ is a special type of operator known as a Fourier integral operator. We refer to [Zw] for background. In [Z5] it was pointed out that the symplectic maps underlying unitary Fourier integral operators can be multivalued correspondences, and that all of the wellknown counter-examples of Sunada [Su] were conjugate by unitary Fourier integral operators (the unitary conjugation was also observed by Bérard, who termed them transplantations). Thus, "most" of the counterexamples are Fourier-isospectral but there are some examples which are not. 
As discussed in $§ 4.3$, one of the main approaches to inverse results is through Birkhoff canonical forms, which are special "local" unitary Fourier integral conjugations between Laplacians in small neighborhoods of closed geodesics. This is a different kind of condition from local isometry of the metrics but is related to it in special cases. See [Gor, Sch] for discussion of isospectral pairs which are not locally isometric.

As this discussion indicates, a natural direction for future work in inverse spectral theory is to remove the symmetry and analyticity assumptions as much as possible. It would be useful to know if (or to what extent) symmetry or analyticity were a spectral invariant for some reasonable classes of metrics, potentials or domains. The only result of this kind known to the author is the recent one of [DHV], which shows that, in the case of the 1-parameter $\hbar$ inverse spectral problem, a radial monotonic potential $V_{0}$ of a Schrödinger operator (2) on $\mathbb{R}^{n}$ is spectrally determined among "all" smooth potentials (not assuming any symmetry of the latter).

With this background in mind we now present the basic objects in inverse spectral theory.

\section{Spectral Invariants}

The data from which one hopes to recover the metric or potential is the list $\left\{\lambda_{j}^{2}\right\}_{j=0}^{\infty}$ of eigenvalues, enumerated with multiplicity, i.e. the dimension of the eigenspace (1). We first consider how to assemble the data into useful invariants.

\subsection{Traces and Trace Formulae}

Since the origins of inverse spectral theory, the principal spectral invariants are defined by trace formulae. One assembles the eigenvalue data into generating functions such as the heat trace $\Theta(t):=\sum_{j} e^{-t \lambda_{j}^{2}}$ or wave trace

$$
S(t):=\sum_{j} e^{i t \lambda_{j}}
$$

The simplest way to relate spectrum and geometry is to study the singularities of these spectral functions.

The heat trace is smooth except at $t=0$, and the coefficients of its expansion at $t=0$ were the first spectral invariants to be studied. In dimension 2 , the heat trace has the form $\Theta(t)=t^{-1} \operatorname{Area}(M, g)+$ $\frac{2 \pi}{3} \chi(M)+\frac{t}{60} \int_{M} \tau_{g}^{2} d V_{g}+\cdots$. There is just one singular term and its coefficient is the integral of a local geometric invariant. One also sees that all of the Taylor coefficients of $t \Theta(t)$ are given by integrals of local geometric invariants. This shows that there are useful spectral invariants besides the ones which arise from singularities.

\subsection{Length Spectrum and Wave Trace Singularities}

The length spectrum of a boundaryless manifold $(M, g)$ is the set

$$
\operatorname{Lsp}(M, g)=\left\{L_{\gamma_{1}}<L_{\gamma_{2}}<\cdots\right\}
$$

of lengths of closed geodesics $\gamma_{j}$, i.e. the set of distinct lengths, not including multiplicities. One refers to the the length spectrum repeated according to multiplicity as the extended length spectrum.

For a generic metric on a manifold without boundary, the length spectrum is a discrete set and moreover the lengths have multiplicity one [SS]. In the boundary case, the length spectrum $\operatorname{Lsp}(\Omega)$ is the set of lengths of closed billiard trajectories, which has accumulation points at lengths of closed billiard trajectories which glide for some interval of time along the boundary. In the case of convex plane domains, e.g., the length spectrum is the union of the lengths of periodic reflecting rays and multiples of $|\partial \Omega|$.

\subsection{Singular Support of the Wave Trace}

The wave group of $(M, g)$ is the unitary group

$$
U(t)=e^{i t \sqrt{-\Delta_{g}}}: L^{2}(M) \rightarrow L^{2}(M) .
$$

Its trace (3) is a distribution on $\mathbb{R}$. The first result on the wave trace is the Poisson relation on a manifold without boundary, stating that the singular support of $S(t)$ is contained in the length spectrum,

$$
\text { Sing Supp } S(t) \subset \operatorname{Lsp}(M, g) \text {. }
$$

It was proved by Y. Colin de Verdière [CdV3], Chazarain [Ch2], and Duistermaat-Guillemin [DG] (following non-rigorous work of Balian-Bloch [BB2] and Gutzwiller). The generalization to manifolds with boundary was proved by Anderson-Melrose [AM] and Guillemin-Melrose [GM]. As above, we denote the length of a closed geodesic $\gamma$ by $L_{\gamma}$. For each $L=L_{\gamma} \in$ $\operatorname{Lsp}(M, g)$ there are at least two closed geodesics of that length, namely $\gamma$ and $\gamma^{-1}$ (its time reversal). The singularities due to these lengths are identical so one often considers the even part of $S(t)$ i.e. $\operatorname{Tr} E(t)$ where

$$
E(t)=\cos \left(t \sqrt{-\Delta_{g}}\right) .
$$

The containment relation (6) could be strict if a length $L \in \operatorname{Lsp}(M, g)$ is multiple. In this case, $S(t)$ might be smooth at $L \in L s p(M, g)$.

One cannot determine multiplicities of lengths from the singularities of $S(t)$ in any simple way. The Sunada-type isospectral pairs [Su] always have multiple length spectra, and for many (presumably, generic) examples, the length spectra have different multiplicities. 
In the notation for $L s p(M, g)$ we wrote $L_{\gamma_{j}}$ as if the closed geodesics of this length were isolated. But in many examples (e.g. spheres or flat tori), the geodesics come in families, and the associated length $T$ is the common length of closed geodesics in the family. In place of closed geodesics, one has components of the fixed point sets of $G^{T}$ at this time.

\subsection{Singularity Expansions}

The next fact is that $S(t)$ has a singularity expansion at each $L \in L \operatorname{sp}(M, g)$ :

$$
S(t) \equiv e_{0}(t)+\sum_{L \in L s p(M, g)} e_{L}(t) \bmod C^{\infty},
$$

where $e_{0}, e_{L}$ are Lagrangean distributions with singularities at just one point, i.e. sing supp $e_{0}=$ $\{0\}$, sing supp $e_{L}=\{L\}$. When the length functional on the loopspace of $M$ is a Bott-Morse functional, the terms have complete asymptotic expansions. In the Morse case (i.e. bumpy metrics), the expansions take the form

$$
\begin{aligned}
e_{0}(t)= & a_{0,-n}(t+i 0)^{-n}+a_{0,-n+1}(t+i 0)^{-n+1}+\cdots \\
e_{L}(t)= & a_{L,-1}(t-L+i 0)^{-1}+a_{L, 0} \log (t-(L+i 0)) \\
& +a_{L, 1}(t-L+i 0) \log (t-(L+i 0))+\cdots
\end{aligned}
$$

where $\cdots$ refers to homogeneous terms of ever higher integral degrees ([DG]). The wave coefficients $a_{0, k}$ at $t=0$ are essentially the same as the singular heat coefficients, hence are given by integrals over $M$ of $\int_{M} P_{j}(R, \nabla R, \ldots) \mathrm{d}$ vol of homogeneous curvature polynomials. The wave invariants for $t \neq 0$ have the form:

$$
a_{L, j}=\sum_{\gamma: L_{\gamma}=L} a_{\gamma, j}
$$

where $a_{\gamma, j}$ depends only on the germ of the metric along $\gamma$. Here, $\{\gamma\}$ runs over the set of closed geodesics, and where $L_{\gamma}, L_{\gamma}^{\#}, m_{\gamma}$, resp. $P_{\gamma}$ are the length, primitive length, Maslov index and linear Poincaré map of $\gamma$. The Poincaré map is defined in (15). For instance, the principal wave invariant at $t=L$ in the case of a non-degenerate closed geodesic is given by

$$
a_{L,-1}=\sum_{\gamma: L_{\gamma}=L} \frac{e^{\frac{i \pi}{4} m_{\gamma}} L_{\gamma}^{\#}}{\left|\operatorname{det}\left(I-P_{\gamma}\right)\right|^{\frac{1}{2}}} .
$$

The same formula for the leading singularity is valid for periodic reflecting rays of compact smooth Riemannian domains with boundary and with Neumann boundary conditions, while in the Dirichlet case the numerator must be multiplied by $(-1)^{r}$ where $r$ is the number of reflection points (see [GM, PS]).

The wave invariants for $t \neq 0$ are both less global and more global than the heat invariants. First, they are more global in that they are not integrals of local invariants, but involve the semi-global first return map of the closed geodesic $\mathcal{P}_{\gamma}$. One could imagine different local geometries producing the same first return map. Second, they are less global because they are determined by the germ of the metric at $\gamma$ and are unchanged if the metric is changed outside $\gamma$.

Thus, associated to any closed geodesic $\gamma$ of $(M, g)$ is the sequence $\left\{a_{\gamma^{r}, j}\right\}$ of wave invariants of $\gamma$ and of its iterates $\gamma^{r}$. These invariants depend only on the germ of the metric at $\gamma$. The principal question of this survey may be stated as follows:

Problem 1. How much of the local geometry of the metric $g$ at $\gamma$ is contained in the wave invariants $\left\{a_{\gamma^{r}, j}\right\}$ ? Can the germ of the metric $g$ at $\gamma$ be determined from the wave invariants? At least, can the symplectic equivalence class of its germ be determined?

As will be discussed below, the classical Birkhoff normal form of the metric (or the Poincaré map $P_{\gamma}$ ) at $\gamma$ is determined by the wave trace invariants [G, G2, $\mathrm{Z3}, \mathrm{Z} 4$ ].

\subsection{Domains and Metrics with the Same Wave Invariants}

Although we are emphasizing wave invariants, it is known that they do not generally determine a domain or metric. There exists an example known as a Penrose mushroom (due to Michael Lifshits) which shows that wave invariants are not sufficient to discriminate between all pairs of smooth billiard tables. Indeed, Lifshitz constructs (many) pairs of smooth domains $\left(\Omega_{1}, \Omega_{2}\right)$ which have the same length spectra and the same wave invariants at corresponding pairs of closed billiard orbits $\gamma_{j}$ of $\Omega_{j}(j=1,2)$. We refer to [FK] for proof that the domains with the same wave invariants in general have no eigenvalues in common. One can prove this by contradiction using a deformation argument; see also [GH] for the generalization to Schrödinger operators.

The domains are constructed in part from ellipses, which have very special billiard dynamics, and are thus far from generic. They are also not real analytic. Thus they exemplify the strange behavior that can result in wildly non-generic cases mentioned in (D) of §0.2. It would be interesting to find spectral invariants that distinguish them.

\subsection{Residual Spectral Invariants and the Poisson-Wave Trace}

More generally one can consider zeta functions such as

$$
S(t, z)=\sum_{j=1}^{\infty} \lambda_{j}^{-z} e^{i t \lambda_{j}}, \quad(\operatorname{Re} z>>0)
$$


and study the poles and residues of its analytic continuation in $z \in \mathbb{C}$. The residual spectral invariants are the residues at the poles. They have local geometric expressions, which relate spectrum and geometry. In particular the wave invariants above are residues of $S(t, z)$ at its poles in $z$ for $t \neq 0$. Note that (3) is the boundary value of the holomorphic function

$$
S(t+i \tau):=\sum_{j} e^{i(t+i \tau) \lambda_{j}}, \quad \tau>0
$$

in the upper half plane $\{t+i \tau: \tau>0\}$. More generally one may define the two-variable holomorphic function $S(t+i \tau, z)=\sum_{j} \lambda_{j}^{-z} e^{i(t+i \tau) \lambda_{j}}$, and study its (branched) meromorphic continuation from $\mathbb{C}_{+} \times$ $\mathbb{C}_{+}(\operatorname{Re} \tau>0, \operatorname{Re} z>>0)$ to $\mathbb{C} \times \mathbb{C}$. There may exist new spectral invariants from this analytic continuation.

\subsection{Non-Residual or Non-Local Spectral Invariants}

There exist many results on such non-local spectral invariants as the lowest eigenvalue $\lambda_{1}^{2}$ or the Laplace determinant det $\Delta_{g}$. One can also consider special values of $\Theta(t)$ or $S(t, z)$ at non-singular values. Non-local invariants are rarely computable in geometric terms. Examples which are the coefficients of positive integral powers of $t$ in the heat trace $\operatorname{Tr} e^{-t \Delta_{g}}$ at $t=0$. In this survey we concentrate on residual spectral invariants.

\section{Explicit Formulae for Wave Invariants}

To explain what is meant by "local geometry" we now give the formula for the "wave trace invariants" or (equivalently) Birkhoff canonical form invariants around a closed geodesic $\gamma$ in the two-dimensional case.

\subsection{The Principal Wave Trace Invariant}

A lot of information about $(M, g)$ is already contained in the principal wave invariant (12). In [DG] (and its Appendix) it is proved that when $\operatorname{Lsp}(M, g)$ is simple, one may recover the lengths of all closed geodesics and the eigenvalues of all of the Poincaré maps $P_{\gamma}$. This suggests already that the geodesic flows of isospectral Riemannian manifolds should not be far from symplectically equivalent in the sense discussed in §0.2, at least in neighborhoods in $S_{g}^{*} M$ of closed geodesics.

\subsection{Riemannian Manifolds Without Boundary}

The wave invariants at a closed geodesic $\gamma$ are invariants of the germ of the metric at $\gamma$. We introduce
Fermi normal coordinates $(s, y)$ along $\gamma$ and denote the corresponding metric coefficients by $g_{i j}$. That is, the coordinates $(s, y)$ correspond to $\exp _{\gamma(s)} y v_{s}$ where $v_{s}$ is the unit normal at the point $\gamma(s)$.

We then consider orthogonal Jacobi fields along $\gamma$, i.e. normal fields $Y(s)=y(s) v_{s}$ where $Y$ solves the Jacobi equation $Y^{\prime \prime}+R\left(Y, \gamma^{\prime}\right) \gamma^{\prime}=0$ along $\gamma$. The space of complex Jacobi fields along $\gamma$ is denoted $\mathcal{J}_{\gamma}$. The linear Poincare map is the monodromy map

$$
P_{\gamma}: Y(t) \rightarrow Y\left(t+L_{\gamma}\right)
$$

on this space. We refer to its eigenvectors $Y_{j}, \bar{Y}_{j}$ as Jacobi eigenvectors.

We assume that the geodesic is non-degenerate in the sense that $\left(\operatorname{det}\left(I-P_{\gamma}\right) \neq 0\right)$. To simplify the exposition, we only consider the case where $\gamma$ is elliptic, i.e. where $P_{\gamma}$ has unit modulus eigenvalues $e^{i \alpha_{j}}$. We define the Floquet invariants

$$
\beta_{j}=\left(1-e^{i \alpha_{j}}\right)^{-1} .
$$

There is a similar story for hyperbolic closed geodesics.

Theorem 2.1. [Z4] Let $\gamma$ be a strongly non-degenerate closed geodesic. Then the kth wave invariant at $\gamma$ has the form

$$
a_{\gamma k}=\int_{\gamma} I_{\gamma ; k}(s ; g) d s
$$

where:

(i) $I_{\gamma ; k}(s ; g)$ is a homogeneous polynomial of weight $-k-1$ (under the scaling $g \rightarrow \varepsilon^{2} g$ ) in the data $\left\{y, \dot{y}, D_{s, y}^{m} g\right\}$ with $m=\left(m_{1}, \ldots, m_{n+1}\right)$ satisfying $|m| \leq$ $2 k+4$

(ii) The degree of $I_{\gamma ; k}$ in the Jacobi field $Y=y v$ is at most $6 k+6$;

(iii) At most $2 k+1$ indefinite integrations over $\gamma$ occur in $I_{\gamma ; k}$;

(iv) The degree of $I_{\gamma ; k}$ in the Floquet invariants $\beta_{j}$ is at most $k+2$.

The formula is simplest in dimension 2, where there is only one Floquet invariant $\beta$. We use the notation $\tau$ for the scalar curvature, $\tau_{v}$ for its unit normal derivative along $\gamma, \tau_{v v}$ for the Hessian $\operatorname{Hess}(\tau)(v, v)$. We denote by $Y$ the unique normalized Jacobi eigenvector along $\gamma$ and by $\dot{Y}$ its time-derivative. The subprincipal wave invariant $a_{\gamma 0}$ is then given by:

$$
a_{\gamma 0}=\frac{a_{\gamma,-1}}{L^{\#}}\left[B_{\gamma 0 ; 4}\left(2 \beta^{2}-\beta-\frac{3}{4}\right)+B_{\gamma 0 ; 0}\right]
$$

where:

(a) $a_{\gamma,-1}$ is the principal wave invariant (12);

(b) $L^{\#}$ is the primitive length of $\gamma, \sigma$ is its Morse index; $P_{\gamma}$ is its Poincaré map; 
(c) $B_{\gamma 0 ; j}$ has the form:

$$
\begin{aligned}
B_{\gamma 0 ; j}= & \frac{1}{L^{\#}} \int_{o}^{L^{\#}}\left[a|\dot{Y}|^{4}+b_{1} \tau|\dot{Y} \cdot Y|^{2}+b_{2} \tau \operatorname{Re}(\bar{Y} \dot{Y})^{2}\right. \\
& \left.+c \tau^{2}|Y|^{4}+d \tau_{v v}|Y|^{4}+e \delta_{j 0} \tau\right] d s \\
& +\frac{1}{L^{\#}} \sum_{0 \leq m, n \leq 3 ; m+n=3} C_{1 ; m n} \frac{\sin ((n-m) \alpha)}{\left|\left(1-e^{i(m-n) \alpha}\right)\right|^{2}} \\
& \times\left|\int_{o}^{L^{\#}} \tau_{v}(s) \bar{Y}^{m} \cdot Y^{n}(s) d s\right|^{2} \\
& +\frac{1}{L^{\#}} \sum_{0 \leq m, n \leq 3 ; m+n=3} C_{2 ; m n} \operatorname{Im}\left\{\int_{o}^{L^{\#}} \tau_{v}(s) \bar{Y}^{m} \cdot Y^{n}(s)\right. \\
& \left.\times\left[\int_{o}^{s} \tau_{v}(t) \bar{Y}^{n} \cdot Y^{m}(t) d t\right] d s\right\}
\end{aligned}
$$

for various universal coefficients. The coefficients $a, b_{1}, b_{2}, c, d, C_{j ; m n}$ are universal and can be determined from computable special cases. A somewhat computable case is that where $\gamma$ is a rotationally invariant geodesic of length $L$ of a surface of revolution, in which case $\tau, \tau_{v}, \tau_{v v}$ are constants and $y=e^{\frac{2 \pi i s}{L}}$ (we assume here that $\tau \equiv 1$ on $\gamma$ ).

If we iterate $\gamma$ (i.e. consider $\gamma^{k}$, traversing $\gamma k$ times), the Floquet invariants become independent and the two $B_{\gamma 0 ; j}(j=0,4)$ are independent. Thus each is a spectral invariant. This is a general phenomenon [G, G2, Z3]. The coefficients $B_{\gamma 0 ; j}$ are Birkhoff canonical form invariants.

\subsection{Bounded Smooth Plane Domains}

Euclidean plane domains $\Omega$ are quite a different problem because the metric is flat (and known) and it is the boundary which is unknown. We express it locally as a graph $y=f(x)$ over the $x$-axis. The role of closed geodesics is played by periodic trajectories of the biliiard flow on $\Omega$. In particular we consider "twobounce" or bouncing ball orbits, which are straight line segments hitting the boundary orthogonally at both endpoints. They always exist if the domain has an isometric involution or if it is convex.

Rather than giving a formula for the subprincipal wave invariant, we give the top order terms (in derivatives of the boundary) for every wave invariant. Modulo terms involving $\leq 2 j-2$ derivatives, the wave trace invariants take the form

$$
\begin{aligned}
B_{\gamma^{r}, j-1} & \\
= & (4 L r) \mathcal{A}_{r}(0) i^{j-1}\left\{2\left(w_{\mathcal{G}_{1, j}^{2 j, 0}}\right)\left(h_{2 r}^{11}\right)^{j} f^{(2 j)}(0)\right. \\
& +4\left(w_{\mathcal{G}_{2, j+1}^{2 j-1,3,0}}\right)\left(h_{2 r}^{11}\right)^{j} \frac{1}{2-2 \cos \alpha / 2}\left(f^{(3)}(0) f^{(2 j-1)}(0)\right) \\
& \left.+4\left(w_{\widehat{\mathcal{G}}_{2, j+1}^{2 j-1,3,0}}\right)\left(h_{2 r}^{11}\right)^{j-2} \sum_{q=1}^{2 r}\left(h_{2 r}^{1 q}\right)^{3}\left(f^{(3)}(0) f^{(2 j-1)}(0)\right)\right\} .
\end{aligned}
$$

Here

- $h_{2 r}^{p q}$ are the matrix elements of the inverse of the Hessian $H_{2 r}$ of the length function $\mathcal{L}$ in suitable (Cartesian graph) coordinates at $\gamma^{r}$;

- $\mathcal{A}_{r}(0)$ is an $\Omega$-independent (non-zero) constant obtained from amplitude of the principal terms at the critical bouncing ball orbit.

- $w_{\mathcal{G}_{1, j}^{2 j 0}}$ (etc.) are certain non-zero combinatorial constants associated to certain Feynman diagrams (graphs).

In this case, the Floquet exponents are buried in the Hessian matrix elements. A higher dimensional generalization of this formula is given in [HZ2].

\section{Inverse Results}

We now give an idea of how one can prove inverse spectral results using the wave trace invariants.

\subsection{Plane Domains with an Up-Down Symmetry}

\subsubsection{Domains with One Symmetry}

The class $\mathcal{D}_{1, L}$ consists of simply connected realanalytic plane domains $\Omega$ satisfying:

- (i) There exists an isometric involution $\sigma$ of $\Omega$;

- (ii) $\sigma$ "reverses" a non-degenerate bouncing ball orbit $\gamma \rightarrow \gamma^{-1}$

- (iii) The lengths $2 r L$ of all iterates $\gamma^{r}$ have multiplicity one in $\operatorname{Lsp}(\Omega)$, and the eigenvalues of the linear Poincare map $P_{\gamma}$ are not roots of unity;

Let $\operatorname{Spec}(\Omega)$ denote the spectrum of the Laplacian $\Delta_{\Omega}$ of the domain $\Omega$ with Dirichlet boundary conditions.

Theorem 3.1. Spec: $\mathcal{D}_{1, L} \mapsto \mathbb{R}_{+}^{\mathbf{N}}$ is 1-1.

Here, $\operatorname{Lsp}(\Omega)$ denotes the length spectrum, i.e. the set of lengths of closed trajectories of the billiard flow of $\Omega$ (see $\S 2$ for backgroun on billiards). By a bouncing ball orbit $\gamma$ is meant a 2-link periodic trajectory of the billiard flow. It corresponds to a line segment in the interior of $\Omega$ which intersects $\partial \Omega$ orthogonally at both boundary points. By rotating and translating $\Omega$ we may assume that $\gamma$ is vertical and that $A=\left(0,-\frac{L}{2}\right)$. In a strip $T_{\varepsilon}(\overline{A B})$, we may locally express $\partial \Omega=\partial \Omega^{+} \cup \partial \Omega^{-}$ as the union of two graphs over the $x$-axis, namely

$$
\begin{aligned}
& \partial \Omega^{+}=\left\{y=f_{+}(x), \quad x \in(-\varepsilon, \varepsilon)\right\}, \\
& \partial \Omega^{-}=\left\{y=f_{-}(x), \quad x \in(-\varepsilon, \varepsilon)\right\} .
\end{aligned}
$$

The symmetry assumptions (i)-(ii) is that $f_{+}(x)=$ $-f_{-}(x)$. Condition (iii) on the multiplicity of $2 L$ means that just the two orbits $\gamma, \gamma^{-1}$ have length $2 L$. In the elliptic case, its eigenvalues $\left\{e^{ \pm i \alpha}\right\}$ are of modulus one 
and we are requiring that $\frac{\alpha}{2 \pi} \notin \mathbb{Q}$. In the hyperbolic case, its eigenvalues $\left\{e^{ \pm \lambda}\right\}$ are real and they are never roots of unity in the non-degenerate case. These are generic conditions in the class of analytic domains.

Corollary 3.2. Let $\mathcal{D}$ be the class of real analytic plane domains with an isometric involution $\sigma$ satisfying:

- (i) $\sigma$ reverses the shortest closed billiard trajectory $\gamma$;

- (ii) $r L_{\gamma}$ are of multiplicity one in $\operatorname{Lsp}(\Omega)$.

Then Spec: $\mathcal{D} \mapsto \mathbb{R}_{+}^{\mathbf{N}}$ is 1-1.

The proof of the corollary from the Theorem is just to observe that any shortest closed trajectory is automatically a bouncing ball orbit [Gh]. Its length is a spectral invariant.

\subsubsection{Sketch of the Proof}

The key point is determine the $2 j-1$ st and $2 j$ th Taylor coefficients of the curvature at each reflection point from the $j-1$ st wave trace invariant for $\gamma$ and its iterates $\gamma^{r}$. If the domain has the symmetries of an ellipse, one obtains the Taylor coefficients immediately from (17), since the odd Taylor coefficients are zero. On the other hand, there is an obstruction to recovering the Taylor coefficients of $f$ when there is only one symmetry: namely, we must recover two Taylor coefficients $f^{(2 j)}(0), f^{(2 j-1)}(0)$ for each new value of $j$ (the degree of the singularity). This is the principal obstacle to overcome.

The expression (17) for the Balian-Bloch invariants of $\gamma, \gamma^{2}, \ldots$ consists of two types of terms, in terms of their dependence on the iterate $r$. They have a common factor of $2 r L\left(h_{2 r}^{11}\right)^{j-2} \mathcal{A}_{r}(0)$, and after factoring it out we obtain one term

$$
\left(h_{2 r}^{11}\right)^{2}\left\{\left(w_{\mathcal{G}_{1, j}^{2 j, 0}} f^{(2 j)}(0)+\frac{\left(w_{\mathcal{G}_{, j, 1}^{2 j-1,0,0}}\right)}{2-2 \cos \alpha / 2} f^{(3)}(0) f^{(2 j-1)}(0)\right\}\right.
$$

which depends on the iterate $r$ through the coefficient $\left(h_{2 r}^{11}\right)^{2}$, and one

$$
\left(w_{\widehat{\mathcal{G}}_{2, j+1}^{2 j-1,3,0}}\right)\left(\sum_{q=1}^{2 r}\left(h_{2 r}^{1 q}\right)^{3} f^{(3)}(0) f^{(2 j-1)}(0)\right)
$$

which depends on $r$ through the cubic sums $\sum_{q=1}^{2 r}\left(h_{2 r}^{1 q}\right)^{3}$ of inverse Hessian matrix elements $h_{2 r}^{p q}$. In order to "decouple" the even and odd derivatives, it suffices to show that the functions $\left(h_{2 r}^{11}\right)^{2}$ and $\sum_{q=1}^{2 r}\left(h_{2 r}^{1 q}\right)^{3}$ are, at least for "most" Floquet angles $\alpha$, linearly independent as functions of $r \in \mathbb{Z}$, i.e. that $\left(h_{2 r}^{11}\right)^{-2} \sum_{q=1}^{2 r}\left(h_{2 r}^{1 q}\right)^{3}$ is a non-constant function of $r$. It is convenient to use the parameter $a=-2 \cos \frac{\alpha}{2}$ and we write the dependence as $h_{2 r}^{i j}(a)$.
We therefore define the "bad" set of Floquet angles by

(19) $\mathcal{B}=\left\{a\right.$ : the sequence $\left\{\left(h_{2 r}^{11}(a)\right)^{-2} \sum_{q=1}^{2 r}\left(h_{2 r}^{1 q}(a)\right)^{3}\right.$, $r=1,2,3, \ldots\}$ is constant in $r\}$.

Using facts about the finite Fourier transform and circulant matrices, we compute that $\mathcal{B}=\{0,1, \pm 2\}$. For Floquet angles outside of $\mathcal{B}$, we can determine all Taylor coefficients $f_{+}^{(j)}(0)$ from the wave invariants and hence the analytic domain.

\subsection{A Higher Dimensional Inverse Result}

It turns out that one can generalize the result to higher dimensions [HZ2] as long as the domain has $\mathbb{Z}_{2}$ symmetries along all coordinate axes. The $(\mathbb{Z} / 2 \mathbb{Z})^{n}$ symmetries are the maps

$$
\sigma_{j}:\left(x_{1}, \ldots, x_{n}\right) \rightarrow\left(x_{1}, \ldots,-x_{j}, x_{j+1}, \ldots, x_{n}\right) .
$$

The symmetry assumption implies that the intersections of the coordinate axes with $\Omega$ are projections of bouncing ball orbits preserved by the symmetries.

We denote by $\mathcal{D}_{L}$ the class of all bounded realanalytic domains such that:

(i) $\sigma_{j}: \Omega \rightarrow \Omega$ is an isometry for all $j=$ $1, \ldots, n$;

(ii) one of the coordinate axis bouncing ball orbits called $\gamma$, is of length $2 L$

(iii) the lengths $2 r L$ of all iterates $\gamma^{r}$ $(r=1,2,3, \ldots)$ have multiplicity one in $\operatorname{Lsp}(\Omega)$;

(iv) $\gamma$ is non-degenerate, i.e. 1 is not an eigenvalue of its Poincaré map $P_{\gamma}$; if $\gamma$ is elliptic and $\left\{e^{ \pm i \alpha_{1}}, \ldots e^{ \pm i \alpha_{n-1}}\right\}$ are the eigenvalues of $P_{\gamma}$, we further require that $\left\{\alpha_{1}, \ldots, \alpha_{n-1}\right\}$ are linearly independent over $\mathbb{Q}$. We assume the same independence condition in the Hyperbolic case or mixed cases.

Multiplicity one means that there exists precisely one closed billiard trajectory of the given length up to time reversal. Let $\operatorname{Spec}_{B}(\Omega)$ denote the spectrum of the Laplacian $\Delta_{\Omega}^{B}$ of the domain $\Omega$ with boundary conditions $B$ (Dirichlet or Neumann).

Theorem 3.3. [HZ2] For Dirichlet (or Neumann) boundary conditions $B$, the map $\operatorname{Spec}_{B}: \mathcal{D}_{L} \rightarrow \mathbb{R}_{+}^{\mathbf{N}}$ is 1-1.

In other words, if two bounded real analytic domains $\Omega_{1}, \Omega_{2} \subset \mathbb{R}^{n}$ possessing the symmetries of an ellipsoid and satisfying the non-degeneracy and length assumptions of (21) have the same Dirichlet (resp. Neumann) spectra, then they are isometric. 
To our knowledge, the only prior positive result on the inverse spectral problem for higher dimensional bounded domains is that a domain with the Dirichlet (or Neumann) spectrum of a ball must be a ball.

The proof of Theorem 3.3 is similar in nature to the proof of Theorem 3.1 but uses the analysis in [He] of the much more complicated dependence on Floquet eigenvalues in higher dimensions.

\subsection{Spectral Determination of Simple Surfaces of Revolution}

A result which is somewhat parallel to Theorem 3.3 is that convex analytic surfaces of revolution (satisfying a non-degeneracy hypothesis) are determined by their spectra (in this class). The profile curve of the surface of revolution is analogous to the "top half" of the boundary of the plane domain, and the $S^{1}$ symmetry is analogous to the symmetry of the top and bottom halves.

The precise class of metrics we consider are those metrics $g$ on $S^{2}$ which belong to the class $\mathcal{R}^{*}$ of real analytic, rotationally invariant metrics on $S^{2}$ with simple length spectrum in the above sense and satisfying the following "simplicity" condition

- $g=d r^{2}+a(r)^{2} d \theta^{2}$

- $\exists ! r_{0}: a^{\prime}\left(r_{0}\right)=0$

- The Poincare map $\mathcal{P}_{0}$ of $r=r_{0}$ is elliptic of twist type.

Convex analytic surfaces of revolution are examples, but there are others. The unique isolated closed geodesic (at distance $r_{0}$ ) is an elliptic orbit.

Theorem 3.4. ([Z3]) Suppose that $g_{1}, g_{2}$ are two real analytic metrics in the class $\mathcal{R}^{*}$ on $S^{2}$, i.e. $\left(S^{2}, g_{i}\right)$ are simple surfaces of revolution for which the meridian geodesic length is simple. Then $\operatorname{Sp}\left(\Delta_{g_{1}}\right)=\operatorname{Sp}\left(\Delta_{g_{2}}\right)$ implies $g_{1}=g_{2}$.

We discuss the proof in $\S 4.4$. We note that the same kind of result should hold for Schrödinger operators (2) for fixed $\hbar=1$, where one metric $g \in \mathcal{R}^{*}$ is fixed and an $S^{1}$-invariant potential $V$ is varied. However, the "simplicity" assumption probably needs to be placed on $V$ and is probably similar to the monotonicity assumption in [DHV].

\subsection{Isospectral Deformations and Spectral Rigidity}

As mentioned in the introduction, one may at least heuristically consider the map $S p$ on the space of metrics, potentials, or domains. The derivative of $S p$ is the map $\delta g \rightarrow\left(\delta \lambda_{1}, \delta \lambda_{2}, \ldots\right)$ from the tangent space to metrics, potentials or domains to sequenes. One may consider variations of the spectral functions, wave invariants etc. Some early works on variations of eigenvalues are [Be, BE, U].
If $\gamma$ is an isolated, non-degenerate closed geodesic of $g$, then for any deformation $g_{t}$ of $g, \gamma$ deforms smoothly as a closed geodesic $\gamma_{t}$ of $g_{t}$ and one may define its variation

$$
\dot{L_{\gamma}}=\left.\frac{d}{d t}\right|_{t=0} L_{\gamma_{t}} .
$$

It is not hard to compute that

$$
\dot{L_{\gamma}}=\int_{\gamma} \dot{g} d s,
$$

where $\dot{g}$ is viewed as a quadratic function on $T M$ and $\gamma$ is viewed as the curve $\left(\gamma(t), \gamma^{\prime}(t)\right)$ in $T M$.

It follows that whenever the closed geodesics are non-degenerate and of multiplicity one in $\operatorname{Lsp}(M, g)$, an isospectral deformation preserves lengths and so

$$
\int_{\gamma} \dot{g} d s=0, \quad \forall \gamma .
$$

In [GK] and in many subsequent articles, it is shown that there cannot exist such a deformation among negatively curved surfaces. The proof is that there does not exist a (non-trivial) symmetric tensor $\dot{g}$ satisfying (24). To our knowledge the analogous result for domains with boundary is unknown. The analogue of a negatively curved surface is a hyperbolic billiard table. Hyperbolicity refers to the Anosov property of the billiard flow. Such a table could be the complement of a disc (or more than one disc) in a negatively curved surface, or a plane domain whose boundary consists of a finite number of concave curves meeting at corners.

Problem 2. Can one generalize the result of $[G K]$ to hyperbolic billiards? Does a hyperbolic billiard table admit isospectral deformations?

\section{Geometric Calculation of Spectral Invariants}

In this section, we briefly explain how one calculates the wave trace invariants explicitly in terms of geometric invariants or (in the boundary case) in terms of the boundary defining function.

\subsection{Parametrix "Path Integral" Versus Canonical Hamiltonian Approach}

Roughly speaking, one has two approaches to constructing the "propagator" $U(t)=e^{-i t \sqrt{\Delta_{g}}}$ in quantum mechanics and studying its trace. One is the "Hamiltonian approach" or "Birkhoff canonical forms" approach in which one constructs the propagator locally around a closed geodesic $\gamma$ by adapting the canonical formalism (creation/annihilation operators, harmonic oscillators, etc.) to a tubular neighborhood of $\gamma$. The canonical formalism is essentially 
the representation theory of the Heisenberg algebra and the symplectic algebra on $L^{2}\left(\mathbb{R}^{n}\right)$. It is not obvious that one can "transplant" the canonical formalism onto a Riemannian manifold, but this is what the theory of quantum Birkhoff normal forms achieves. Its origins lie in constructions of Gaussian beams by Babich, Lazutkin, Ralston and others (see [BB]). Although Gaussian beams only exist for stable elliptic closed geodesics, the Birkhoff canonical form construction works locally around any non-degenerate closed geodesic (and even around degenerate ones).

The second approach is the Lagrangian (or "path integral") approach in which one constructs a parametrix for the wave group. Unlike the canonical forms approach, there is nothing canonical about the parametrix construction. It is an art to construct a parametrix well adapted to a given problem. The parametrix approach is the traditional one and the reader is probably familiar with the heat kernel parametrix (if not with the term "parametrix").

Let us review the original wave kernel parametrix for (7) due to J. Hadamard (1921). On a Riemannian manifold without boundary, one can construct a Hadamard parametrix for $\cos t \sqrt{\Delta}(x, x)$ for small times, i.e. an approximation for the Schwartz kernel $E(t, x, y)$ of the solution operator of the Cauchy problem,

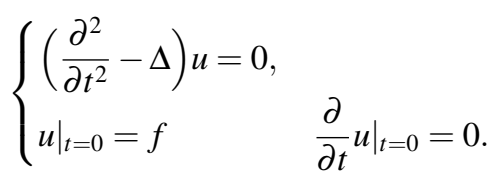

The parametrix is an oscillatory integral (26)

$$
E(t, x, y) \sim \int_{0}^{\infty} e^{i \theta\left(r^{2}-t^{2}\right)} \sum_{j=0}^{\infty} U_{j}(x, y) \theta_{\text {reg }}^{\frac{n-1}{2}-j} d \theta \bmod C^{\infty}
$$

where the Hadamard-Riesz coefficients $U_{j}$ are determined inductively by the transport equations

$$
\begin{aligned}
& \frac{\Theta^{\prime}}{2 \Theta} U_{0}+\frac{\partial U_{0}}{\partial r}=0 \\
& 4 i r(x, y)\left\{\left(\frac{k+1}{r(x, y)}+\frac{\Theta^{\prime}}{2 \Theta}\right) U_{k+1}+\frac{\partial U_{k+1}}{\partial r}\right\}=\Delta_{y} W U_{k} .
\end{aligned}
$$

The solutions are given by:

$$
\begin{aligned}
& U_{0}(x, y)=\Theta^{-1 / 2}(x, y) \\
& U_{j+1}(x, y)=\Theta^{-1 / 2}(x, y) \int_{0}^{1} s^{j} \Theta\left(x, x_{s}\right)^{1 / 2} \Delta_{2} U_{j}\left(x, x_{s}\right) d s
\end{aligned}
$$

where $x_{s}$ is the geodesic from $x$ to $y$ parametrized proportionately to arc-length and where $\Delta_{2}$ operates in the second variable. Also, $r^{2}(x, y)$ is the distance squared, $d V_{g}=\Theta(x, y) d y$ in normal coordinates centered at $x$, and $\theta_{\text {reg }}^{\frac{n-1}{2}-j}$ refers to the regularization of this distribution by analytic continuation (M. Riesz).
The parametrix is a "geometric construction" of the wave group and one may determine the singularities of the wave trace by expressing it in terms of the parametrix. The Hadamard parametrix above is useful to obtain the singularity at $t=0$ and contains the information of the heat trace there. But the above parametrix is only valid for small $t$ since it uses $r^{2}(x, y)$ and $\Theta(x, y)$. To obtain information at much longer times such as $t \in \operatorname{Lsp}(M, g)$ one needs to construct a parametrix which is valid at that time around a closed geodesic. There are a number of clever ways to do this but none are simple except in special cases such as manifolds with no conjugate points. In that case one may follow the method of Selberg (1956) to construct the wave kernel on the universal cover and summing over the deck transformation group (see [Ber, Z1] for references and background).

\subsection{Domains with Boundary}

In the case of bounded domains, it is difficult to construct a parametrix that is valid in the glancing region of geodesics tangent to the boundary. A microlocal parametrix for the Dirichlet or Neumann even wave group $E_{B}^{\Omega}(t)=\cos t \sqrt{\Delta_{B}}$ near a transversal reflecting ray was constructed by J. Chazarain [Ch] (see also [GM, PS] for more details).

To prove Theorem 3.1, the author used the construction of the wave group (or rather, Green's function) using the method of layer potentials as in BalianBloch [BB1, BB2] rather than using a parametrix. This method is used universally in the physics literature to make calculations of eigenvalues and eigenfunctions and it is natural to use it for inverse spectral problems.

We fix a non-degenerate bouncing ball orbit $\gamma$ of length $L_{\gamma}$, and let $\hat{\rho} \in C_{0}^{\infty}\left(L_{\gamma}-\varepsilon, L_{\gamma}+\varepsilon\right)$ be a cutoff, equal to one on an interval $\left(L_{\gamma}-\varepsilon / 2, L_{\gamma}+\varepsilon / 2\right)$ which contains no other lengths in $\operatorname{Lsp}(\Omega)$ occur in its support. The wave trace invariants associated to $\gamma$ are the coefficients of the asymptotic expansion as $k \rightarrow \infty$ of

$$
R_{\rho B}^{\Omega}(k+i \tau)=\int_{0}^{\infty} \hat{\rho}(t) e^{i(k+i \tau) t} E_{B}^{\Omega}(t) d t .
$$

When $\gamma, \gamma^{-1}$ are the unique closed orbits of length $L_{\gamma}$, it follows from the Poisson relation for manifolds with boundary (see $\S 3$ and [GM]) that (29) has the expansion

(30)

$$
\operatorname{Tr} 1_{\Omega} R_{\rho}(k+i \tau) \sim e^{(i k-\tau) L_{\gamma}} \sum_{j=1}^{\infty}\left(B_{\gamma, j}+B_{\gamma^{-1}, j}\right) k^{-j}, \quad k \rightarrow \infty
$$

with coefficients $B_{\gamma, j}, B_{\gamma^{-1}, j}$ determined by the jet of $\Omega$ at the reflection points of $\gamma$. The coefficients $B_{\gamma, j}, B_{\gamma^{-1}, j}$ are thus essentially the same as the wave trace coefficients at the singularity $t=L_{\gamma}$. 
As mentioned above, it is the art in inverse spectral theory to find a method by which the coefficients can be calculated explicitly. One might use a well-chosen parametrix around $\gamma$ or use a canonical form. In [Z4], we use the layer potential method of [BB1, BB2] to express the Dirichlet (resp. Neumann) resolvent in terms of the "free resolvent" $R_{0}(k+i \tau)=$ $-\left(\Delta_{0}+(k+i \tau)^{2}\right)^{-1}$ on $\mathbb{R}^{2}$, in the form,

\section{(31)}

$$
\begin{aligned}
R_{\Omega}(k+i \tau)= & R_{0}(k+i \tau) \\
& -\mathcal{D} \ell(k+i \tau)(I+N(k+i \tau))^{-1} \gamma \mathcal{S} \ell^{t r}(k+i \tau) .
\end{aligned}
$$

Here, $\gamma: H^{s}(\Omega) \rightarrow H^{s-1 / 2}(\partial \Omega)$ is the restriction to the boundary, and $\mathcal{D} \ell(k+i \tau)$ (resp. $\mathcal{S} \ell(k+i \tau)$ ) is the double (resp. single) layer potential, i.e. the operator from $H^{s}(\partial \Omega) \rightarrow H_{l o c}^{s+1 / 2}(\Omega)$ defined by

$$
\left\{\begin{array}{l}
\mathcal{S} \ell(k+i \tau) f(x)=\int_{\partial \Omega} G_{0}(k+i \tau, x, q) f(q) d s(q) \\
\mathcal{D} \ell(k+i \tau) f(x)=\int_{\partial \Omega} \frac{\partial}{\partial v_{y}} G_{0}(k+i \tau, x, q) f(q) d s(q)
\end{array}\right.
$$

where $d s(q)$ is the arc-length measure on $\partial \Omega$, where $v$ is the interior unit normal to $\Omega$. Also, $\mathcal{S} \ell^{t r}$ is its transpose (from the interior to the boundary), and $G_{0}(z, x, y)$ is the free Green's function, i.e. the kernel of $R_{0}(z)$. Further,

(33) $N(k+i \tau) f(q)=2 \int_{\partial \Omega} \frac{\partial}{\partial v_{y}} G_{0}\left(k+i \tau, q, q^{\prime}\right) f\left(q^{\prime}\right) d s\left(q^{\prime}\right)$

is the boundary integral operator induced by $\mathcal{D} \ell$. One may simplify the trace by combining the interior and exterior problems. We write $L^{2}\left(\mathbb{R}^{2}\right)=L^{2}(\Omega) \oplus L^{2}\left(\Omega^{c}\right)$ and let $R_{N}^{\Omega}$, resp. $(k+i \tau), R_{D}^{\Omega^{c}}(k+i \tau)$ denote the Neumann resolvent on the exterior domain, resp. the Dirichlet resolvent on the interior domain. We then regard $R_{D}^{\Omega^{c}}(k+i \tau) \oplus R_{N}^{\Omega}(k+i \tau)$ as an operator on this space. We cycle around the layer potentials in (31) when taking the trace to obtain,

$$
\begin{aligned}
& \operatorname{Tr}_{\mathbb{R}^{2}}\left[R_{D}^{\Omega^{c}}(k+i \tau) \oplus R_{N}^{\Omega}(k+i \tau)-R_{0}(k+i \tau)\right] \\
& \quad=\frac{d}{d k} \log \operatorname{det}(I+N(k+i \tau))
\end{aligned}
$$

where the determinant is the usual Fredholm determinant. The main point then is to prove the existence of the following asymptotic expansion:

Proposition 4.1. Suppose that $L_{\gamma}$ is the only length in the support of $\hat{\rho}$. Then,

$$
\int_{\mathbb{R}} \rho(k-\lambda) \frac{d}{d \lambda} \log \operatorname{det}(I+N(\lambda+i \tau)) d \lambda \sim \sum_{j=0}^{\infty} B_{\gamma ; j} k^{-j},
$$

where $B_{\gamma ; j}$ are the wave invariants of $\gamma$.
One then shows that $N(k+i \tau)$ is a semi-classical Fourier integral operator quantizing the billiard map on the boundary. Let us explain these terms. The billiard map $\beta: B^{*} \partial \Omega \rightarrow B^{*} \partial \Omega$ on the ball bundle of $\partial \Omega$ is defined as follows: given $(y, \eta) \in B^{*} \partial \Omega$, with $|\eta|<1$ we let $(y, \zeta) \in S^{*} \Omega$ be the unique inward-pointing unit covector at $y$ which projects to $(y, \eta)$ under the map $T_{\partial \Omega}^{*} \Omega \rightarrow T^{*} \partial \Omega$. Then we follow the geodesic (straight line) determined by $(y, \zeta)$ to the first place it intersects the boundary again; let $y^{\prime} \in Y$ denote this first intersection, and let $\eta^{\prime}$ be the projection of $\zeta$ to $B_{y^{\prime}}^{*} \partial \Omega$. Then we define

$$
\beta(y, \eta)=\left(y^{\prime}, \eta^{\prime}\right)
$$

We say that $N(k+i \tau)$ quantizes the billiard map in that its Schwartz kernel is (almost) given by

$$
N\left(k+i \tau, y, y^{\prime}\right) \sim(k+i \tau)^{(n-2)} e^{i(k+i \tau)\left|y-y^{\prime}\right|} \tilde{b}\left(k\left|y-y^{\prime}\right|\right),
$$

where $\tilde{b}(t)$ has an expansion in inverse powers of $t$ as $t \rightarrow \infty$, with leading term $\sim t^{(-n+1) / 2}$. This is proved in [HaZe, Z4]. The kernel is a semiclassical FIO of order 0 as long as $\left|y-y^{\prime}\right|>k^{-\frac{1}{2}} \log k$. We separate out the tangential and transversal parts of $N$ by introducing a cutoff of the form $\chi\left(k^{1-\delta}\left|q-q^{\prime}\right|\right)$ to the diagonal, where $\delta>1 / 2$ and where $\chi \in C_{0}^{\infty}(\mathbb{R})$ is a cutoff to a neighborhood of 0 . We then put

$$
\left\{\begin{array}{l}
N_{0}\left(k+i \tau, q, q^{\prime}\right)=\chi\left(k^{1-\delta}\left|q-q^{\prime}\right|\right) N\left(k+i \tau, q, q^{\prime}\right), \\
N_{1}\left(k+i \tau, q, q^{\prime}\right)=\left(1-\chi\left(k^{1-\delta}\left|q-q^{\prime}\right|\right)\right) N\left(k+i \tau, q, q^{\prime}\right) .
\end{array}\right.
$$

The term $N_{1}$ is a semiclassical Fourier integral kernel quantizing the billiard map, while $N_{0}$ behaves like an Airy operator close to the diagonal with the singularity of a homogeneous pseudodifferential operator on the diagonal.

The advantage of this approach over the canonical forms method or using a Chazarain (or other parametrix) is that the kernel $N\left(k, q, q^{\prime}\right)$ is independent of the boundary except for the restriction of the layer potential to the given boundary. In some sense the expansion above is "canonical". The phase $\left|y-y^{\prime}\right|$ on $\partial \Omega \times \partial \Omega$ is the generating function of the billiard map as long as $\Omega$ is convex in the sense of symplectic geometry. If $\Omega$ fails to be convex, it generates a canonical relation which is larger than the graph of $\beta$, causing some technical problems that can be handled [HaZe]. One also must take into account the part of the kernel very close to the diagonal, which is particularly important if one is studying billiard trajectories that creep along the boundary but it is not important if one considers trajectories that are transverse to the boundary such as bouncing ball orbits. 
At least formally, we then expand $(I+N(k+i \tau))^{-1}$ in a finite geometric series plus remainder:

$$
\begin{aligned}
(I+N(\lambda+i \tau))^{-1}= & \sum_{M=0}^{M_{0}}(-1)^{M} N(\lambda)^{M} \\
& +(-1)^{M_{0}+1} N(\lambda)^{M_{0}+1}(I+N(\lambda+i \tau))^{-1}
\end{aligned}
$$

We then write

$$
\text { (38) }\left(N_{0}+N_{1}\right)^{M}=\sum_{\sigma:\{1, \ldots, M\} \rightarrow\{0,1\}} N_{\sigma(1)} \circ N_{\sigma(2)} \circ \cdots \circ N_{\sigma(M)} \text {. }
$$

We regularize $N^{M}$ by eliminating the factors of $N_{0}$ from each of these terms. This is obviously not possible for the term $N_{0}^{M}$ but it is possible for the other terms. By explicitly writing out the composition in terms of Hankel functions and using the basic identities for these special functions, we prove that $N_{0} \circ$ $\left.N_{1} \circ \chi_{0}\left(k+i \tau, \varphi_{1}, \varphi_{2}\right)\right)$ is a semiclassical Fourier integral operator on $\partial \Omega$ of order -1 associated to the billiard map. Thus, composition with $N_{0}$ lowers the order. The remaining terms $N_{0}^{M}$, when composed with a cut-off to $\gamma$, do not contribute asymptotically to the trace.

The successive removal of the factors of $N_{0}$ thus gives a semi-classical quantization of the billiard map near $\gamma$. We then calculate the traces of each term by the stationary phase method to obtain the expansion (30). The coefficients containing the top derivatives for each power of $k$ are rather straightforward to calculate explicitly.

\subsection{Birkhoff Canonical Form}

So far we have described parametrix methods for evaluating wave trace coefficients. We now describe the Birkhoff canonical form and the sense in which it is canonical. Let $\gamma$ be a non-degenerate closed geodesic on an $n$-dimensional Riemannian manifold, and at first let us assume it to be elliptic. For each transversal quantum number $q \in \mathbb{Z}^{n-1}$, there was an approximate eigenvalue of the form

$$
\lambda_{k q} \equiv r_{k q}+\frac{p_{1}(q)}{r_{k q}}+\frac{p_{2}(q)}{r_{k q}^{2}}+\ldots
$$

where

$$
r_{k q}=\frac{1}{L}\left(2 \pi k+\sum_{j=1}^{n}\left(q_{j}+\frac{1}{2}\right) \alpha_{j}\right)
$$

where the coefficients are polynomials of specified degrees and parities. We refer to [BB] for a clear exposition and for details.

A natural question is whether the wave invariants $a_{\gamma j}$ of (10) can be determined from the quasieigenvalues (39), i.e. from the polynomials $p_{j}(q)$. The answer is "yes" and in effect (39) is the canonical form.

To put $\Delta$ into normal form, is first to conjugate it into a distinguished maximal abelian algebra $\mathcal{A}$ of pseudodifferential operators on a model space, the cylinder $S_{L}^{1} \times \mathbb{R}^{n}$, where $S_{L}^{1}$ is the circle of length $L$. The algebra is generated by the tangential operator $D_{s}:=\frac{\partial}{i \partial s}$ on $S_{L}^{1}$ together with the transverse action operators. The nature of these action operators depends on $\gamma$. When $\gamma$ is elliptic, the action operators are harmonic oscillators

$$
I_{j}=I_{j}\left(y, D_{y}\right):=\frac{1}{2}\left(D_{y_{j}}^{2}+y_{j}^{2}\right),
$$

while in the real hyperbolic case they have the form

$$
I_{j}=y_{j} D_{y_{j}}+D_{y_{j}} y_{j} .
$$

When $\gamma$ is non-degenerate, they involve some mixture of these operators (and also complex hyperbolic actions) according to the spectral decomposition of $P_{\gamma}$. For notational simplicity we restrict to the elliptic case and put

$$
H_{\alpha}:=\frac{1}{2} \sum_{k=1}^{n} \alpha_{k} I_{k}
$$

where $e^{ \pm i \alpha_{k}}$ are the eigenvalues of the Poincare map $P_{\gamma}$.

To put $\Delta$ into normal form is to conjugate it to the model space and algebra as a function of $D_{s}$ and the action operators. The conjugation is only defined in a neighborhood of $\gamma$ in $T^{*} M-0$, i.e. one constructs a microlocally elliptic Fourier Integral operator $W$ from the conic neighborhood of $\mathbb{R}^{+} \gamma$ in $T^{*} N_{\gamma}-0$ to a conic neighborhood of $T_{+}^{*} S_{L}^{1}$ in $T^{*}\left(S_{L}^{1} \times R^{n}\right)$ such that:

$$
\begin{aligned}
W \sqrt{\Delta_{\psi}} W^{-1} \equiv & \mathcal{D}+\frac{\tilde{p}_{1}\left(\hat{I}_{1}, \ldots, \hat{I}_{n}\right)}{L \mathcal{D}}+\frac{\tilde{p}_{2}\left(\hat{I}_{1}, \ldots, \hat{I}_{n}\right)}{(L \mathcal{D})^{2}}+\ldots \\
& +\frac{\tilde{p}_{k+1}\left(\hat{I}_{1}, \ldots, \hat{I}_{n}\right)}{(L \mathcal{D})^{k+1}}+\ldots
\end{aligned}
$$

where the numerators $p_{j}\left(\hat{I}_{1}, \ldots, \hat{I}_{n}\right), \tilde{p}_{j}\left(\hat{I}_{1}, \ldots, \hat{I}_{n}\right)$ are polynomials of degree $\mathrm{j}+1$ in the variables $\hat{I}_{1}, \ldots, \hat{I}_{n}$, where $W^{-1}$ denotes a microlocal inverse to $W$. Here, $\mathcal{D}=$ $D_{s}+\frac{1}{L} H_{\alpha}$. The $k$ th remainder term lies in the space $\oplus_{j=o}^{k+2} O_{2(k+2-j)} \Psi^{1-j}$, where $\Psi^{s}$ denotes the pseudodifferential operators on the model space of order $s$ and where $O_{2(k+2-j)}$ denotes the operators whose symbols vanish to the order $2(k+2-j)$ along $\gamma$. We observe that (40) is an operator version of (39).

The inverse result of [G], see also [Z3, Z4]] is:

Theorem. Let $\gamma$ be a non-degenerate closed geodesic. Then the quantum Birkhoff normal form around $\gamma$ is a spectral invariant; in particular the classical Birhoff normal form is a spectral invariant. 
In other words, one can determine the polynomials $p_{j}\left(\hat{I}_{1}, \ldots, \hat{I}_{n}\right)$ from the wave trace invariants of $\Delta$ at $\gamma$. The key point is that one can construct the normal form explicity by re-scaling the metric around $\gamma$ and making a step-by-step conjugating to Hermite operators and creation/annihilation operators first define by Babich, Lazutkin and others (see [BB] for background). Thus, the algorithm is quite concrete and leads to computable spectra invariants of Theorem 2.1. But to date, the formulae have not been applied to any concrete inverse spectral problems due to their complexity. In [Z2, HZ2] we found that inverse results could be proved in the boundary case by just keeping track of the highest order derivative terms in the wave invariants. It is possible that the argument could be generalized to some metric cases.

\subsection{Proof of Theorem 3.4 on Spectral Determination of Analytic Simple Surfaces of Revolution}

As mentioned above, surfaces of revolution are the surfaces most analogous to bounded plane domains in that both are defined by a profile function of one real variable. This suggests that one should be able to prove an analogue of Theorem 3.1 for analytic surfaces of revolution. In fact, it was proved first and by a normal forms method. To our knowledge, it remains the only inverse spectral result for metrics which was proved by using Birkhoff normal forms, and therefore we briefly recall the method. Recently, similar methods have been used in the $\hbar$ potential problem for (2) in several settings [CdVG, CdV2, GW, GU].

The proof is based on quantum Birkhoff normal forms for the Laplacian $\Delta$. But the special feature of simple surfaces of revolution is that there exists a global Birkhoff normal form as well as local ones around the critical closed orbit or the invariant tori. It was constructed in [CdV4] and expresses the fact tht $\Delta$ is a toric integrable Laplacian in the following sense: there exist commuting first order pseudo-differential operators $\hat{I}_{1}, \hat{i}_{2}$ such that:

- the joint spectrum is integral, i.e. $\operatorname{Sp}(\mathcal{L}) \subset \mathbb{Z}^{2} \cap \Gamma+$ $\{\mu\}$ where $\Gamma$ is the cone $I_{2} \geq\left|I_{1}\right|$ in $\mathbb{R}^{2}$.

- The square root of $\Delta$ is a first order polyhomogenous function $\sqrt{\Delta}=\hat{H}\left(\hat{I}_{1}, \hat{I}_{2}\right)$ of the action operators.

By polyhomogeneous, we mean that $\hat{H}$ has an asymptotic expansion in homogeneous functions of the form:

$$
\hat{H} \sim H_{1}+H_{-1}+\ldots, \quad H_{j}(r I)=r^{j} H_{j}(I) .
$$

The principal symbols $I_{j}$ of the $\hat{I}_{j}$ 's generate a classical Hamiltonian torus action on $T^{*} S^{2}-0$. There is no term of order zero. It follows that

$$
S p\left(\sqrt{\Delta_{g}}\right)=\left\{\hat{H}(N+\mu): N \in \mathbb{Z}^{2} \cap \Gamma_{o}\right\},
$$

where the eigenvalues have expansions

$$
\lambda_{N} \sim H_{1}(N+\mu)+H_{-1}(N+\mu)+\ldots .
$$

Theorem 4.2. ([Z2]) Let $\left(S^{2}, g\right)$ be an analytic simple surface of revolution with simple length spectrum. Then the normal form $\hat{H}\left(\xi_{1}, \xi_{2}\right)$ is a spectral invariant.

The normal form and the proof are very different from the non-degenerate case in [G, Z3, Z4]. Recently, similar kinds of results have been proved in other integrable settings [CPV, ChV].

To complete the proof of Theorem 3.4, we need to show that $\hat{H}$ determines a metric in $\mathcal{R}$. As in the bounded domain case outline above, we need to calculate the normal form invariants. It turns out to be sufficient to calculate $H_{1}=H$ and $H_{-1}$ in terms of the metric (i.e. in terms of $a(r)$ ) and then to invert the expressions to determine $a(r)$. In [Z2] $H$ and $H_{-1}$ were calculaed using by studying asymptotics $\sqrt{\Delta}=$ $\hat{H}\left(\hat{I}_{1}, \hat{I}_{2}\right)$ along "rays of representations" of the quantum torus action, i.e. along multiples of a given lattice point $\left(n_{0}, k_{0}\right)$. In effect, the $n_{0}$ parameter is like $\hbar^{-1}$ and we reduce to a one-dimensional Sturm-Liouville problem. Thus, Theorem 4.2 converts the hard problem into the easier problem of determining the potential for semi-classical Schrödinger operators. In a rather standard way, one finds that

$$
\int_{\mathbb{R}}(E-x)_{+}^{\frac{1}{2}} d \mu(x)
$$

is a spectral invariant, where $\mu$ is the distribution function $\mu(x):=\left|\left\{r: \frac{1}{a(r)^{2}} \leq x\right\}\right|$ of $\frac{1}{a^{2}}$, with $|\cdot|$ the Lebesgue measure. This Abel transform is invertible and hence

$$
d \mu(x)=\sum_{r: \frac{1}{a(r)^{2}}=x}\left|\frac{d}{d r} \frac{1}{a(r)^{2}}\right|^{-1} d x
$$

and therefore

$$
J(x):=\sum_{r: a(r)=x} \frac{1}{\left|a^{\prime}(r)\right|}
$$

are spectral invariants. By the simplicity assumption on $a$, there are just two solutions of $a(r)=x$; the smaller will be written $r_{-}(x)$ and the larger, $r_{+}(x)$. Thus, the function

$$
J(x)=\frac{1}{\left|a^{\prime}\left(r_{-}(x)\right)\right|}+\frac{1}{\left|a^{\prime}\left(r_{+}(x)\right)\right|}
$$

is a spectral invariant. 
By studying $H_{-1}$, we find in a somewhat similar way that

$$
K(x)=\left|a^{\prime}\left(r_{-}(x)\right)\right|+\left|a^{\prime}\left(r_{+}(x)\right)\right|
$$

is a spectral invariant. It follows that we can determine $a^{\prime}\left(r_{+ \pm}(x)\right)$.

Since both metrics $g_{1}$ and $g_{2}$ are assumed to belong to $\mathcal{R}^{*}$, they are determined by their respective functions $a_{j}(r)$. We conclude that $a_{1}=a_{2}$ and hence that $g_{1}=g_{2}$.

In more recent work on the inverse problem for the $\hbar$-spectra of Schrödinger operators, the methods are similar to the final step in the proof above but allow potentials which have more critical points. It is then a challenge to recover the potential from its distribution function and the higher invariants. Viceversa, the methods might be used for more general inverse problems for metrics on surfaces of revolution in which one relaxes the simplicity assumption.

\section{Isospectral Deformations of Domains}

An isospectral deformation of a plane domain $\Omega_{0}$ is a one-parameter family $\Omega_{\varepsilon}$ of plane domains for which the spectrum of the Euclidean Dirichlet (or Neumann) Laplacian $\Delta_{\varepsilon}$ is constant (including multiplicities). We say that $\Omega_{\varepsilon}$ is a $C^{1}$ curve of $C^{\infty}$ plane domains if there exists a $C^{1}$ curve of diffeomorphisms $\varphi_{\varepsilon}$ of a neighborhood of $\Omega_{0} \subset \mathbb{R}^{2}$ with $\varphi_{0}=i d$ and with $\Omega_{\varepsilon}=\varphi_{\varepsilon}\left(\Omega_{0}\right)$. The infinitesimal generator $X=\frac{d}{d \varepsilon} \varphi_{\varepsilon}$ is a vector field in a neighborhood of $\Omega_{0}$ which restricts to a vector field along $\partial \Omega_{0}$; we denote by $X_{v}=\dot{\rho} v$ its outer normal component. With no essential loss of generality we may assume that $\left.\varphi_{\varepsilon}\right|_{\partial \Omega_{0}}$ is a map of the form

$$
x \in \partial \Omega_{0} \rightarrow x+\rho_{\varepsilon}(x) v_{x},
$$

where $\rho_{\varepsilon} \in C^{1}\left(\left[0, \varepsilon_{0}\right], C^{\infty}\left(\partial \Omega_{0}\right)\right), \varepsilon_{0}>0$ and $\rho_{0}=0$. We put $\dot{\rho}(x)=\delta \rho(x):=\left.\frac{d}{d \varepsilon}\right|_{\varepsilon=0} \rho_{\varepsilon}(x)$. An isospectral deformation is said to be trivial if $\Omega_{\varepsilon} \simeq \Omega_{0}$ (up to isometry) for sufficiently small $\varepsilon$. A domain $\Omega_{0}$ is said to be spectrally rigid if all isospectral deformations $\Omega_{\varepsilon}$ are trivial. The domain $\Omega_{0}$ is called infinitesimally spectrally rigid if $\dot{\rho}=0$ (up to rigid motions) for all isospectral deformations.

The variations of the eigenvalues are given by Hadamard's variational formulae,

$$
\dot{\lambda}_{j}(0)= \begin{cases}\left.\int_{\partial \Omega(0)} \rho\left|\partial_{\nu} \varphi_{j}(0)\right|_{\partial \Omega}\right|^{2} d A, & \text { Dirichlet } \\ \left.\int_{\partial \Omega} \rho\left|\varphi_{j}(0)\right|_{\partial \Omega(0)}\right|^{2} d A, & \text { Neumann }\end{cases}
$$

Hence, the infinitesimal deformation condition is that the right hand sides are zero for all $j$. To normalize the problem, we assume with no loss of generality that the deformation is volume preserving, which implies that

$$
\int_{\partial \Omega} \rho d A=0
$$

Any such $\rho$ defines a volume preserving deformation of $\Omega$.

Thus, the infinitesimal deformation is orthogonal to all boundary traces of eigenfunctions:

$$
\dot{\lambda}_{j}(0)=0 \quad \forall j, \Longleftrightarrow\left\{\begin{array}{l}
\left.\int_{\partial \Omega(0)} \rho\left|\partial_{\nu} \varphi_{j}(0)\right|_{\partial \Omega}\right|^{2} d A=0, \\
\text { Dirichlet } \\
\left.\left.\int_{\partial \Omega} \rho\left|\varphi_{j}(0)\right|\right|_{\partial \Omega(0)}\right|^{2} d A=0, \\
\text { Neumann. }
\end{array}\right.
$$

We may rewrite these conditions in terms of the boundary values of the wave kernel:

$$
E^{b}(t, x, x):= \begin{cases}\left.\partial_{v_{x}} \partial_{v_{y}} U(t, x, x)\right|_{x \in \partial \Omega}, & \text { Dirichlet } \\ \left.U(t, x, x)\right|_{x \in \partial \Omega}, & \text { Neumann }\end{cases}
$$

as saying that

$$
\int_{\partial \Omega} E^{b}(t, x, x) \rho(x) d A(x)=0, \quad \forall t .
$$

\subsection{Spectral Rigidity of the Ellipse}

Problem 3. Is an ellipse $E_{a, b}$ given by $\frac{x^{2}}{a^{2}}+\frac{y^{2}}{b^{2}}=1$ determined by its Dirichlet or Neumann spectrum? Is it spectrally rigid, i.e. does there exist a non-trivial isospectral deformation $\Omega_{t}$ of $E_{a, b}$ ? Since the area and perimeter are spectral invariants it is understood that $\Omega_{t}$ has fixed area and perimeter.

In the case of circles, the answer was shown to be "yes" by M. Kac, since the disc is extremal for the isoperimetric inequality. But the answer remains unknown for general ellipses. Ellipses are special because they have completely integrable billiard flows, and (since G. D. Birkhoff) are conjectured to be the only smooth plane domains with integrable billiards.

In [HZ], H. Hezari and the author proved a partial rigidity result which breaks out of the class of real analytic domains, but does assume that the domains all possess the symmetries of the ellipse. Namely, ellipses are spectrally rigid among $C^{\infty}$ plane domains with the symmetries of an ellipse. Here "rigidity" means that there do not exist smooth curves $\Omega_{t}$ in the family of isometry classes of smooth domains with the spectrum of the ellipse, at least if the curves are "non-flat" in that the Taylor expansion of the domain at the endpoins of the axes is non-zero in the deformation variable $t$. This non-flatness assumption is an unexpected and annoying technicality which it would be nice to remove. The result is 
Theorem 5.1. Suppose that $\Omega_{0}$ is an ellipse, and that $\varepsilon \rightarrow \Omega_{\varepsilon}$ is a $C^{\infty}$ Dirichlet (or Neumann) isospectral deformation through $\mathbb{Z}_{2} \times \mathbb{Z}_{2}$ symmetric $C^{\infty}$ domains. Then $\rho_{\varepsilon}$ must be flat at $\varepsilon=0$. In particular, there exist no non-trivial real analytic curves $\varepsilon \rightarrow \Omega_{\varepsilon}$ of $\mathbb{Z}_{2} \times \mathbb{Z}_{2}$ symmetric $C^{\infty}$ domains with the spectrum of an ellipse.

We used the Hadamard variational formula in the proof. By taking the variation of the wave trace, we proved:

Theorem 5.2. Let $\Omega_{0} \subset \mathbb{R}^{n}$ be a $C^{\infty}$ convex Euclidean domain with the property that the fixed point sets of the billiard map are clean. Then, for any $C^{1}$ variation of $\Omega_{0}$ through $C^{\infty}$ domains $\Omega_{\varepsilon}$, the variation of the wave traces $\delta \operatorname{Tr} \cos (t \sqrt{-\Delta}$ ), with Dirichlet (or Neumann) boundary conditions is a classical co-normal distribution for $t \neq m\left|\partial \Omega_{0}\right|(m \in \mathbb{Z})$ with singularities contained in $\operatorname{Lsp}\left(\Omega_{0}\right)$. For each $T \in \operatorname{Lsp}\left(\Omega_{0}\right)$ for which the set $F_{T}$ of periodic points of the billiard map $\beta$ of length $T$ is a d-dimensional clean fixed point set consisting of transverse reflecting rays, there exist nonzero constants $C_{\Gamma}$ independent of $\dot{\rho}$ such that, near $T$, the leading order singularity is

$$
\begin{aligned}
\delta \operatorname{Tr} \cos (t \sqrt{-\Delta}) \sim & \frac{t}{2} \operatorname{Re}\left\{\left(\sum_{\Gamma \subset F_{T}} C_{\Gamma} \int_{\Gamma} \dot{\rho} \gamma_{1} d \mu_{\Gamma}\right)\right. \\
& \left.\times\left(t-T+i 0^{+}\right)^{-2-\frac{d}{2}}\right\},
\end{aligned}
$$

modulo lower order singularities. The sum is over the connected components $\Gamma$ of $F_{T}$. Here $\delta=\left.\frac{d}{d \varepsilon}\right|_{\varepsilon=0}$ and $\gamma_{1}(q, \zeta)=\sqrt{1-|\zeta|^{2}}$.

Here $\gamma_{1}$ is a certain computable function on $B^{*} \partial \Omega$.

The billiard flow and billiard map of the ellipse are completely integrable. In particular, except for certain exceptional trajectories, the periodic points of period $T$ form a Lagrangian tori in $S^{*} \Omega_{0}$. The exceptions are the two bouncing ball orbits through the major/minor axes and the trajectories which intersect the foci or glide along the boundary. The fixed point sets of $\Phi^{T}$ intersect the co-ball bundle $B^{*} \partial \Omega_{0}$ of the boundary in the fixed point sets of the billiard map $\beta: B^{*} \partial \Omega_{0} \rightarrow B^{*} \partial \Omega_{0}$ (for background we refer to [PS, GM, HZ] for instance). Except for the exceptional orbits, the fixed point sets are real analytic curves. For the bouncing ball rays, the associated fixed point sets are non-degenerate fixed points of $\beta$.

The densities $d \mu_{\Gamma}$ on the fixed point sets of $\beta$ and its powers are very similar to the canonical densities defined in Lemma 4.2 of [DG], and further discussed in [GM, PT]. The constants $C_{\Gamma}$ are explicit and depend on the boundary conditions. Also, $F_{T}$ is the curve of periodic points of the billiard map $\beta$ on $B^{*} \partial \Omega$ of length $T$, where as above the length is defined by the length of the corresponding billiard trajectory.
The dimension $d$ is the dimension of $F_{T}$. In the case of the ellipse, for instance, $d=1$; the periodic points of a given length form invariant curves for $\beta$.

Theorem 5.3. Suppose that $\Omega_{0}$ is an ellipse, and that $\Omega_{\varepsilon}$ is a $C^{1}$ Dirichlet (or Neumann) isospectral deformation of $\Omega_{0}$ through $C^{\infty}$ domains with $\mathbb{Z}_{2} \times \mathbb{Z}_{2}$ symmetry. I.e. is invariant under $(x, y) \rightarrow( \pm x, \pm y)$. Then $\dot{\rho}=0$.

This implies that ellipses admit no isospectral deformations for which the Taylor expansion of $\rho_{\varepsilon}$ at $\varepsilon=0$ is non-trivial. A function such as $e^{-\frac{1}{\varepsilon^{2}}}$ for which the Taylor series at $\varepsilon=0$ vanishes is called "flat" at $\varepsilon=0$.

Since the final step of the proof uses results of [GM], we briefly review the description of the billiard map of the ellipse $\Omega_{0}:=\frac{x^{2}}{a}+\frac{y^{2}}{b}=1$ (with $a>b>0$ ) in that article. In the interior, there exist for each $0<Z \leq$ $b$ a caustic set given by a confocal ellipse

$$
\frac{x^{2}}{E+Z}+\frac{y^{2}}{Z}=1
$$

where $E=a-b$, or for $-E<Z<0$ by a confocal hyperbola. Let $(q, \zeta)$ be in $B^{*} \partial \Omega_{0}$ and let $(q, \xi)$ in $S^{*} \Omega_{0}$ be the unique inward unit normal to boundary that projects to $(q, \zeta)$. The line segment $(q, r \xi)$ will be tangent to a unique confocal ellipse or hyperbola (unless it intersects the foci). We then define the function $Z(q, \zeta)$ on $B^{*} \partial \Omega_{0}$ to be the corresponding $Z$. Then $Z$ is a $\beta$-invariant function and its level sets $\{Z=c\}$ are the invariant curves of $\beta$. The invariant Leray form on the level set is denoted $d u_{Z}$ (see [GM], (2.17), i.e. the symplectic form of $B^{*} \partial \Omega_{0}$ is $d q \wedge d \zeta=d Z \wedge d u_{Z}$. A level set has a rotation number and the periodic points live in the level sets with rational rotation number. As it is explained in [GM] (page 143) the Leray form $d u_{Z}$ restricted to a connected component $\Gamma$ of $F_{T}$ is a constant multiple of the canonical density $d \mu_{\Gamma}$.

As mentioned in the introduction, the well-known obstruction to using trace formula calculations such as in Proposition 5.2 is multiplicity in the length spectrum, i.e. existence of several connected components of $F_{T}$. A higher dimensional component is not itself a problem, but there could exist cancellations among terms coming from components with different Morse indices, since the coefficients $C_{\Gamma}$ are complex. This problem arose earlier in the spectral theory of the ellipse in [GM]. Their key Proposition 4.3 shows that there is a sufficiently large set of lengths $T$ for which $F_{T}$ has one component up to $(q, \zeta) \rightarrow(q,-\zeta)$ symmetry. Since it is crucial here as well, we state the relevant part:

Proposition 5.4. (see [GM], Proposition 4.3): Let $T_{0}=$ $\left|\partial \Omega_{0}\right|$. Then for every interval $\left(m T_{0}-\varepsilon, m T_{0}\right)$ for $m=$ $1,2,3, \ldots$ there exist infinitely many periods $T \in$ $\operatorname{Lsp}\left(\Omega_{0}\right)$ for which $F_{T}$ is the union of two invariant 
curves which are mapped to each other by $(q, \zeta) \rightarrow$ $(q,-\zeta)$.

Since for an isospectral deformation $\delta \operatorname{Tr} \cos (t \sqrt{-\Delta})=0$, we obtain from Proposition 5.2 the following

Corollary 5.5. Suppose we have an isospectral deformation of an ellipse $\Omega_{0}$ with velocity $\dot{\rho}$. Then for each $T$ in Proposition 5.4 for which $F_{T}$ is the union of two invariant curves $\Gamma_{1}$ and $\Gamma_{2}$ which are mapped to each other by $(q, \zeta) \rightarrow(q,-\zeta)$ we have

$$
\int_{\Gamma_{j}} \dot{\rho} \gamma_{1} d u_{Z}=0, \quad j=1,2 .
$$

Proof. From Proposition 5.2 we get

$$
\operatorname{Re}\left\{\left(\sum_{j=1}^{2} C_{\Gamma_{j}} \int_{\Gamma_{j}} \dot{\rho} \gamma_{1} d \mu_{\Gamma_{j}}\right)(t-T+i 0)^{-2-\frac{d}{2}}\right\}=0 .
$$

Since $\dot{\rho}$ and $\gamma_{1}$ are invariant under the time reversal map $(q, \zeta) \rightarrow(q,-\zeta)$, the two integrals are identical. Also by directly looking at the stationary phase calculations it can be shown that the Maslov coefficients $C_{\Gamma_{1}}$ and $C_{\Gamma_{2}}$ are also the same. Thus the corollary follows.

\subsection{Abel Transform}

The remainder of the proof of Theorem 5.3 is identical to that of Theorem 4.5 of [GM] (see also [PT]). For the sake of completeness, we sketch the proof.

Proposition 5.6. The only $\mathbb{Z}_{2} \times \mathbb{Z}_{2}$ invariant function $\dot{\rho}$ satisfying the equations of Corollary 5.5 is $\dot{\rho}=0$.

Proof. First, we may assume $\dot{\rho}=0$ at the endpoints of the major/minor axes, since the deformation preserves the $\mathbb{Z}_{2} \times \mathbb{Z}_{2}$ symmetry and we may assume that the deformed bouncing ball orbits will not move and are aligned with the original ones. Thus $\dot{\rho}( \pm \sqrt{a})=$ $\dot{\rho}( \pm \sqrt{b})=0$.

The Leray measure may be explicitly evaluated (see 2.18 in [GM]). By a change of variables with Jacobian $J$, and using the symmetric properties of $\dot{\rho}$, the integrals become

$$
A(Z)=\int_{b}^{a} \frac{\dot{\rho}(t) \gamma_{1} J(t) d t}{\sqrt{t-(b-Z)}} .
$$

for an infinite sequence of $Z$ accumulating at $b$. The function $A(Z)$ is smooth in $Z$ for $Z$ near $b$. It vanishes infinitely often in each interval $(b-\varepsilon, b)$, hence is flat at $b$. The $k$ th Taylor coefficient at $b$ is

$$
A^{(k)}(b)=\int_{b}^{a} \dot{\rho}(t) \gamma_{1} J(t) t^{-k-\frac{1}{2}} d t=0 .
$$

Since the functions $t^{-k}$ span a dense subset of $C[b, a]$, it follows that $\dot{\rho} \equiv 0$.
We refer to $\S 7.1$ for some further problems regarding the isospectral determinantion problem for ellipses.

5.2.1 Spectral Rigidity of Convex Analytic Domain with the Symmetries of an Ellipse?

We observe that the proof of Theorem 5.1 only uses the hypothesis that the domain is an ellipse in two steps. First, in Theorem 5.2 it uses the very special kind of singularities of the wave trace due to the foliation of $B^{*} \partial E$ by invariant curves for the billiard flow. Then in Proposition 5.6 it uses the explicit formula (47) for the integrals over the invariant curves. In the case of general convex smooth domains, there exist invariant sets for the billiard map due to periodic creeping orbits along the boundary, but they are much more complicated than in the completely integrable case of the ellipse. Still, it may be possible to analyze the analogous integrals $A(Z)$.

\section{Inverse Scattering, Phase Shifts, Resonance Poles}

In this section we consider the inverse phase shift problem for semi-classical Schrödinger operators (2) on $\mathbb{R}^{d}$ (with the Euclidean Laplacian). The scattering matrix

$$
S_{h}: L^{2}\left(\mathbb{S}^{d-1}\right) \rightarrow L^{2}\left(\mathbb{S}^{d-1}\right)
$$

can be defined in terms of generalized eigenfunctions as follows. For $\varphi_{i n} \in C^{\infty}\left(\mathbb{S}^{d-1}\right)$, there is a unique solution to $H u=0$ satisfying

$$
\begin{aligned}
u= & r^{-(d-1) / 2}\left(e^{-i \sqrt{E} r / h} \varphi_{\text {in }}(\omega)+e^{i \sqrt{E} r / h} \varphi_{\text {out }}(-\omega)\right) \\
& +O\left(r^{-(d+1) / 2}\right) .
\end{aligned}
$$

By definition

$$
S_{h}\left(\varphi_{\text {in }}\right):=e^{i \pi(d-1) / 2} \varphi_{\text {out }} .
$$

Below, we will refer to $\varphi_{\text {in }}$ and $\varphi_{\text {out }}$ as the incoming and outgoing data of $u$. It is not hard to show that

$$
S_{h, V}(E)=S_{\widetilde{h}, \widetilde{V}}(1),
$$

where $\widetilde{h}=h / \sqrt{E}, \widetilde{V}=V / E$, and $S_{h^{\prime}, V^{\prime}}\left(E^{\prime}\right)$ denotes the scattering matrix for $\left(h^{\prime}\right)^{2} \Delta+V^{\prime}-E^{\prime}$. Using (51), we may assume that $E=1$.

Phase shifts are the eigenvalues for the eigenvalue problem

$$
S_{h} \varphi_{h, n}=e^{i \beta_{h, n}} \varphi_{h, n}
$$

Problem 4. Can one determine the potential $V$ (in some reasonable class) from the phase shifts $\left\{e^{i \beta_{h, n}}\right\}_{n=1}^{\infty}$ for fixed energy but as $h$ varies? 
The simplest problem is to assume maximal symmetry, i.e. that the potential is radial. This effectively reduces the problem to one dimension. The scattering matrix then commutes with the action of $S O(d)$ and $\Delta_{S^{d-1}}$ and thus has just one eigenvalue in each eigenspace of the Laplacian. When $d=3$, the eigenvalues of $\Delta_{S^{2}}$ on the sphere are known as the total angular momenta, and thus the phase shifts are traditionally denoted $\delta_{\ell}(k)$ where the angular momentum is $\ell(\ell+1)$ and $k=h^{-1}$.

The question of finding a radial potential $V$ from its phase shifts has been much studied since the 1960 's. The phase shifts are closely related to the Jost functions $f_{\ell}(k)=\left|f_{\ell}(k)\right| e^{-i \delta_{\ell}(j)}$. The first issue is whether (2) has any $L^{2}$-eigenfunctions or "bound states". In classical scattering the corresponding issue is whether there exist trapped Hamilton trajectories or whether they all escape to infinity. It one knows all of the eigenvalues $E_{n}(\hbar)$ and the phase shifts $\delta_{\ell}(k)$ for all $k \geq 0$ then one can construct $\left|f_{\ell}(k)\right|$. Equivalently one knows the ratio $f_{\ell}(-k) / f_{\ell}(k)$. The problem of determining $f_{\ell}(k)$ from the ratios is a Riemann-Hilbert problem. Using the Gelfand-Levitan solution of the inverse spectral problem in dimension one, it has been shown that if there are $N$ eigenvalues then there exists an N-parameter family of potentials with the same phase shifts as $k$ varies and $\ell$ is fixed and same eigenvalues [N, N2, N3]. In particular, the potential is uniquely determined if (2) has no $L^{2}$ eigenfunctions. If instead one fixes $k$ and lets $\ell$ vary, one can also solve the problem but not uniquely.

To our knowledge, the problem of determining $V$ from the $\beta_{h, n}$ in non-radial cases is open and the analogues of the methods described above for Laplacians on compact manifolds have not been discussed.

A closely related problem is to study the Laplacian $\Delta$ of the Euclidean metric in the exterior of a convex obstacle $\mathcal{O}$. Thus, we let $\Omega=\mathbb{R}^{d} \backslash \mathcal{O}$ and consider the scattering matrix $S_{h}$ for $h^{2} \Delta$ on $L^{2}(\Omega)$ with Dirichlet or Neumann boundary conditions.

Problem 5. Can one determine $\Omega$ from the fixed energy phase shifts $e^{i \beta_{h, n}}$ ?

The inverse problem for obstacles is trivial in the radial case but very non-trivial in general. There exist results on determining the potential from the scattering kernel (Theorem 2 of [Maj1976, G3]), but to our knowledge the phase shift problem has not been studied. The method of [G3] is to determine the socalled sojourn times from the full scattering kernel $S_{h}(\omega, \theta)$ as $h \rightarrow 0$ and to show that it dtermines the Gaussian curvature of the surface. In the case of a convex obstacle, the curvature function determines the obstacle, i.e. the shape of the scatterer. The question of how much of the shape of the scatterer one might recover from the phase shifts alone does not seem to have been studied in the non-radial case.

As J. Gell-Redman emphasized to the author, the so-called interior-exterior duality establishes a relation between the interior Dirichlet eigenvalues and the exterior Dirichlet phase shifts [EP]. From the phase shifts one can determine the interior Dirichlet eigenvalues. Hence positive results on the inverse problem for domains imply positive results for phase shifts.

We refer to [Maj1976, ChS, CT, KKS, MT, N, N2, N3, Sab] for background on inverse results in dimensions $>1$ and to [GHZ, DGHH2013] for some recent results on the distribution of phase shifts. Some further references in dimension one are given in the introduction.

\section{Problems and Partial Solutions}

In this section we state some problems which are at least partly open. They are intended both to give a sense of the current state of the art and also to suggest problems on which it seems likely one can make progress.

The following is probably the most natural:

Problem 6. Is a convex analytic domain spectrally rigid in the class $\mathcal{C} \mathcal{A}$ of convex smooth domains? More difficult: Is a $C^{\infty}$ convex domain spectrally rigid?

There are several possible approaches. One is to develop the proof of Theorem 5.1. Only in the last step is the geometry of the ellipse used. It will change radically for a general convex domain. In particular one must use the invariant sets which arise in general.

In view of the number of inverse spectral results which use the assumption that the domains are real analytic, we pose:

Problem 7. Suppose $\Omega_{1}$ and $\Omega_{2}$ are isospectral plane domains. If $\Omega_{1}$ is real analytic, is $\Omega_{2}$ real analytic? Is this true if we assume both domains have the symmetry of an ellipse?

The reason for posing the problem is that, when a domain has the symmetry of an ellipse, one can determine the Taylor expansion of the domain at the endpoints of the two axes from the spectrum. Hence if the expansion converges for the first domain, the Taylor series converges for the second domain. But it might not actually equal the second domain. More precisely, we represent the top and bottom of the domains as graphs $y=f_{ \pm}(x)$ over one of the axes and then the Taylor expansion of $f_{ \pm}^{j}(x) j=1,2$ is determined by the spectrum. Under the symmetry assumption $f_{-}^{j}=-f_{+}^{j}$. For instance, in the case of an ellipse $E_{a, b}, f_{+}(x)=b \sqrt{1-\frac{x^{2}}{a^{2}}}$. We note that the radius of convergence of $f_{ \pm}$at $x=0$ is $|x|<a$, i.e. until the graph 
ceases to be a graph by turning vertical. The length of the axes is a spectral invariant and therefore it must coincide with the first domain.

The question arises whether $\Omega_{2}$ is locally defined by $f_{ \pm}^{1}$. It is possible that $f_{ \pm}^{2}=f_{ \pm}^{1}+\psi_{ \pm}$where $\psi$ vanishes to infinite order at the endpoints of the bouncing ball orbit. The wave invariants still coincide but it is not clear that the wave trace itself does. One might need "exponentially small corrections" to the wave trace to determine that, or else a proof that the wave trace expansion converges. It is not clear that this is possible for isospectral surfaces. A simpler problem is

Problem 8. Suppose $\Omega_{1}$ and $\Omega_{2}$ are isospectral plane domains. If $\Omega_{1}$ is real analytic, is $\Omega_{2}$ locally real analytic at the endpoints of a bouncing ball orbit? Is this true if we assume both domains have the symmetry of an ellipse?

If so, we then ask: Suppose that $\Omega_{2}$ is isospectral to a real analytic $\Omega_{1}$ and that $\partial \Omega_{2}=\partial \Omega_{1}$ on some interval $x \in(-\varepsilon, \varepsilon)$ around the endpoints of a bouncing ball orbit. Must then $\Omega_{2}=\Omega_{1}$ ?

Another question concerns the normal form in the analytic case.

Problem 9. Does the quantum Birkhoff normal form at a hyperbolic geodesic $\gamma$ converge if the metric is real analytic? Does the conjugating map (unitary operator) converge?

It is proved in [Mo] that this is true for the classical Birkhoff normal forms in dimension 2. Some hints that the answer is positive for quantum normal forms in dimension 2 are given in $[\mathrm{Sj}, \mathrm{A}, \mathrm{Sa}, \mathrm{PM}, \mathrm{I}]$. If the answer is "yes" then the manifolds are locally Fourierisospectral near $\gamma$. In particular the geodesic flows are locally symplectically equivalent.

Problem 10. Do isospectral plane domains have symplectically equivalent billiard maps? If a domain $\Omega$ has the same eigenvalues as the ellipse $E_{a, b}$, does it have a completely integrable billiard map?

Results of Siburg [S] and of the author and G. Forni show that the billiard flow would be $C^{0}$ conjugate to that of the ellipse, at least if one has an isospectral deformation. But there is a long distance between $C^{0}$ and smooth conjugacy.

\subsection{Questions About Oscillatory Integrals}

Problem 11. If an oscillatory integral $\int_{\mathbb{R}^{n}} a e^{i k \varphi} d x$ whose phase has a single critical point at $x=0$ has the stationary phase asymptotics of a Morse function with a single critical point, is it a Morse function? Similarly, if it has the stationary phase asymptotics of a BottMorse phase function with a non-degenerate critical manifold, is it Bott-Morse?
The reason for posing this problem is that wave trace invariants are obtained by applying stationary phase to a parametrix for the wave group, and one only knows the wave trace invariants explicitly if the closed geodesics are non-degenerate (i.e. if the phase of the integral is Morse) or "clean" (if the phase is Bott-Morse). If a second domain or metric has the same wave trace invariants as the first, then its wave trace expansion at a closed geodesic is the same as one for first, given by stationary phase for a Morse function. It would simplify the inverse problem considerably if we could know that that the closed geodesic of the unknown metric or domain was also Morse (or Bott-Morse). For instance in the case of the ellipse, the phase functions are Bott-Morse.

As a potential application, we pose:

Problem 12. Suppose that $\Omega$ is isospectral to an ellipse. Do the periodic orbits of the billiard map $\beta$ on $B^{*} \partial \Omega$ form smooth invariant curves? Similarly, if $\left(S^{2}, g\right)$ is a metric on $S^{2}$ which is isospectral to a convex surface of revolution, do the periodic geodesics form smooth invariant Lagrangian tori?

The point is that the wave trace invariants are the same as for the ellipse. resp. $\left(S^{2}, g\right)$ (here we ignore issues of length spectral multiplicity). As we see from Theorem 5.2, the wave trace has the singularities of a Bott-Morse oscillatory integral with clean fixed point sets of dimension 2 (in $S_{g}^{*} M$ ) resp. 1 (in $B^{*} \partial \Omega$ ). A positive answer to the Problem about oscillatory integrals with the stationary phase asymptotics of a Bott-Morse function would imply that the phase has to be BottMorse for the unknown metric or boundary and therefore would be smooth invariant submanifolds.

A related more ambitious question is:

Problem 13. Suppose that $\Omega$ is isospectral to an ellipse. Is $B^{*} \partial \Omega$ foliated by smooth invariant curves for $\beta$ ? Similarly, if $\left(S^{2}, g\right)$ is a metric on $S^{2}$ which is isospectral to a surface of revolution, is $S_{g}^{*} S^{2}$ foliated by smooth Lagrangian tori invariant under the geodesic flow?

\section{References}

[AM] Z. S. Agranovich and V. A. Marchenko, The inverse problem of scattering theory. Gordon and Breach Science Publishers, New York-London 1963.

[A] V. A. Ambarzumian, Über eine frage der eigenwerttheorie, Z. Phys. 53 (1929), 690-695.

[An] A. Anikin, Normal form of a quantum Hamiltonian with one and a half degrees of freedom near a hyperbolic fixed point. Regul. Chaotic Dyn. 13 (2008), no. 5, 377-402.

[BB] V. M. Babic, V. S. Buldyrev: Short-Wavelength Diffraction Theory, Springer Series on Wave Phenomena 4, Springer-Verlag, New York (1991).

[BB1] R. Balian and C. Bloch, Distribution of eigenfrequencies for the wave equation in a finite domain I: three-dimensional problem with smooth boundary surface, Ann. Phys. 60 (1970), 401-447. 
[BB2] R. Balian and C. Bloch, Distribution of eigenfrequencies for the wave equation in a finite domain. III. Eigenfrequency density oscillations. Ann. Physics 69 (1972), 76-160.

[Bar1] V. Bargmann, Remarks on the determination of a central field of force from the elastic scattering phase shifts. Physical Rev. (2) 75 (1949), 301-303.

[Bar2] V. Bargmann, On the connection between phase shifts and scattering potential. Rev. Modern Physics 21 (1949), 488-493.

[Ber] P. Bérard, Transplantation et isospectralité. I. Math. Ann. 292 (1992), no. 3, 547-559.

[Be] M. Berger, Quelques formules de variation pour une structure riemannienne. Ann. Sci. École Norm. Sup. (4) 3 (1970), 285-294.

[BE] M. Berger, D. Ebin, Some decompositions of the space of symmetric tensors on a Riemannian manifold. J. Differential Geometry 3 (1969), 379-392.

[Bo] G. Borg, Eine Umkehrung der Sturm-Liouvilleschen Eigenwertaufgabe. Bestimmung der Differentialgleichung durch die Eigenwerte. Acta Math. 78 (1946), $1-96$.

[BrH] J. Brüning and E. Heintze, Spektrale Starrheit gewisser Drehflächen. Math. Ann. 269 (1984), no. $1,95-101$.

[ChS] K. Chadan and P. C. Sabatier, Inverse problems in quantum scattering theory. With a foreword by R. G. Newton. Texts and Monographs in Physics. Springer-Verlag, New York-Berlin, 1977.

[CPV] L. Charles, A. Pelayo, and San Vu Ngoc, Isospectrality for quantum toric integrable systems, arXiv:1111.5985.

[ChV] L. Charles and V. N. San, Spectral Asymptotics via the semiclassical Birkhoff Normal Form, Duke Math. J. 143 (2008), 463-511, MR2423760, Zbl 1154.58015. MR2423760 (2009d:58049).

[Ch] J. Chazarain, Construction de la paramétrix du problème mixte hyperbolique pour l'Équation des ondes. C. R. Acad. Sci. Paris Sér. A-B 276 (1973), A1213-A1215.

[Ch2] J. Chazarain, Formule de Poisson pour les variétés riemanniennes. Invent. Math. 24 (1974), 65-82.

[CdV] Y. Colin de Verdière, Sur les longueurs des trajectoires périodiques d'un billard, pp. 122-139, in Séminaire sud-rhodanien de géométrie (Lyon, 1983), vol. 3, edited by P. Dazord and N. DesolneuxMoulis, Hermann, Paris, 1984, MR86a:58078, Zbl 0599.58039.

[CdV2] Y. Colin de Verdière, A Semi-classical Inverse Problem II: Reconstruction of the Potential, Geometric aspects of analysis and mechanics, 97-119, Progr. Math., 292, Birkhäuser/Springer, New York, 2011, MR2809469 (2012e:34022).

[CdV3] Y. Colin de Verdière, Spectre du laplacien et longueurs des géodésiques périodiques. I, II. Compositio Math. 27 (1973), 83-106; ibid. 27 (1973), 159-184.

[CdV4] Y. Colin de Verdière, Spectre conjoint d'opérateurs pseudo-différentiels qui commutent. II. Le cas intégrable. Math. Z. 171 (1980), no. 1, 51-73.

[CdVG] Y. Colin de Verdière and V. Guillemin, A Semiclassical Inverse Problem I: Taylor Expansion, Geometric aspects of analysis and mechanics, 81-95, Progr. Math., 292, Birkhäuser/Springer, New York, 2011, MR2809468 (2012e:34021).

[CT] J. R. Cox and K. W. Thompson, On the inverse scattering problem at fixed energy for potentials having nonvanishing first moments. J. Mathematical Phys. 11 (1970), 805-814
[DGHH2013] K. Datchev, J. Gell-Redman, A. Hassell, and P. Humphries. Approximation and equidistribution of phase shifts: spherical symmetry. Comm. Math. Phys. 326 (2014), no. 1, 209-236.

[DH] K. Datchev and H. Hezari, Inverse problems in spectral geometry, Inside Out II, p. 455-485, Math. Sci. Res. Inst. Publ., 60, Cambridge Univ. Press, Cambridge, 2013.

[DHV] K. Datchev, H. Hezari, and I. Ventura, Spectral uniqueness of radial semiclassical Schrödinger operators. Math. Res. Lett. 18 (2011), no. 3, 521-529.

[DG] J. J. Duistermaat and V. W. Guillemin, The spectrum of positive elliptic operators and periodic bicharacteristics, Invent. Math. 29 (1975), no. 1, 39-79.

[Dy] F. J. Dyson, Fredholm determinants and inverse scattering problems. Comm. Math. Phys. 47 (1976), no. 2, 171-183.

[Dy2] F. J. Dyson, Old and New Approaches to the Inverse Scattering Problem, in Studies in Mathematical Physics, Essays in Honor of Valetine Bargmann, p. 151-167, Ed. E. Lieb, Princeton UP (1976).

[EP] J. P. Eckmann and C. A. Pillet Spectral duality for planar billiards. Comm. Math. Phys. 170 (1995), no. 2, 283-313, MR1334397.

[F] L. D. Faddeev, The inverse problem in the quantum mechanical theory of scattering, Uspekhi Mat. Nauk 14 (1959), 57-119; J. Math. Phys. 4 (1963), 72104.

[FK] S. A. Fulling and P. Kuchment, Coincidence of length spectra does not imply isospectrality. Inverse Problems 21 (2005), no. 4, 1391-1395.

[GL] I. M. Gelfand and B. M. Levitan, On the determination of a differential equation by its spectral function, Izv. Akad. Nauk SSSR ser. Math. 15 (1951), 309-360; Amer. Math. Soc. Transl. (2) 1 (1955), 253-304.

[GHZ] J. Gell-Redman, A. Hassell, and S. Zelditch, Equidistribution of phase shifts in semiclassical potential scattering, to appear in J. London Math. Soc. (arXiv:1311.2353).

[Gh] M. Ghomi, Shortest periodic billiard trajectories in convex bodies. Geom. Funct. Anal. 14 (2004), no. 2, 295-302.

[Gor] C. S. Gordon, Isospectral closed Riemannian manifolds which are not locally isometric, J. Diff. Geom. 37 (1993), 639-649, MR1217163 (94b:58098).

[GP] C. Gordon, P. Perry, and D. Schueth, Isospectral and isoscattering manifolds: a survey of techniques and examples. Geometry, spectral theory, groups, and dynamics, 157-179, Contemp. Math., 387, Amer. Math. Soc., Providence, RI, 2005.

[GWW] C. Gordon, D. Webb and S. Wolpert, Isospectral plane domains and surfaces via Riemannian orbifolds, Invent. Math. 110 (1992), 1-22.

[G] V. Guillemin, Wave-trace invariants. Duke Math. J. 83 (1996), no. 2, 287-352.

[G2] V. Guillemin, Wave-trace invariants and a theorem of Zelditch. Internat. Math. Res. Notices (1993), no. 12, 303-308

[G3] V. Guillemin, Sojourn times and asymptotic properties of the scattering matrix. Proceedings of the Oji Seminar on Algebraic Analysis and the RIMS Symposium on Algebraic Analysis (Kyoto Univ., Kyoto, 1976). Publ. Res. Inst. Math. Sci. 12 (1976/77), supplement, 69-88.

[GH] V. Guillemin and H. Hezari, A Fulling-Kuchment theorem for the 1D harmonic oscillator. (English summary) Inverse Problems 28 (2012), no. 4, 045009, $9 \mathrm{pp}$. 
[GK] V. Guillemin and D. Kazhdan, Some inverse spectral results for negatively curved 2-manifolds. Topology 19 (1980), no. 3, 301-312.

[GM] V. Guillemin and R. Melrose, An inverse spectral result for elliptical regions in R2, Adv. in Math. 32:2(1979), 128-148.

[GU] V. Guillemin and A. Uribe, Some inverse spectral results for semi-classical Schrödinger operators, Math. Res. Lett. 14 (2007), no. 4, 623-632.

[GW] V. Guillemin and Z. Wang, Semiclassical spectral invariants for Schrödinger operators. J. Differential Geom. 91 (2012), no. 1, 103-128.

[HaZe] A. Hassell and S. Zelditch, Quantum ergodicity of boundary values of eigenfunctions. Comm. Math. Phys. 248 (2004), no. 1, 119-168.

[He] H. Hezari, Inverse spectral problems for Schrödinger operators. Comm. Math. Phys. 288 (2009), no. 3, 1061-1088.

[HZ] H. Hezari and S. Zelditch, $C^{\infty}$ spectral rigidity of the ellipse. Anal. PDE 5 (2012), no. 5, 1105-1137.

[HZ2] H. Hezari and S. Zelditch, S Inverse spectral problem for analytic (Z/2Z)n-symmetric domains in $\mathbb{R}^{n}$. Geom. Funct. Anal. 20 (2010), no. 1, 160-191.

[Hy] E. A. Hylleraas, Calculation of a perturbing central field of force from the elastic scattering phase shift. Physical Rev. (2) 74 (1948), 48-51.

[I] H. Ito, Convergence of Birkhoff normal forms for integrable systems, Comment. Math. Helv. 64 (1989), 412-461.

[KKS] J. B. Keller, I. Kay, and J. Shmoys. Determination of the potential from scattering data, Phys. Rev. (2) 102 (1956), 557-559.

[L] N. Levinson, The inverse Sturm-Liouville problem. Mat. Tidsskr. B. (1949), 25-30.

[Lo] J. Loeffel, On an inverse problem in potential scattering theory. Ann. Inst. H. Poincaré Sect. A (N.S.) 8 (1968), 339-447.

[Maj1976] A. Majda. High frequency asymptotics for the scattering matrix and the inverse problem of acoustical scattering, Comm. Pure Appl. Math. 29 (1976), no. 3, 261-291.

[Mar] V. A. Marchenko, V. On reconstruction of the potential energy from phases of the scattered waves. (Russian) Dokl. Akad. Nauk SSSR (N.S.) 104 (1955), 695-698.

[Mar2] V. A. Marchenko, The generalized shift, transformation operators, and inverse problems, in Mathematical Events of the Twentieth Century, eds. A. A. Bolibruch et al. (Springer, Berlin, 2006).

[MM] S. Marvizi and R. Melrose, Spectral invariants of convex planar regions, J. Differential Geom. 17 (1982), no. 3, 475-502. MR85d:58084, Zbl 0492.53033.

[MT] M. Mijatović and K. Trenčevski. Classical inverse problem for finite scattering region. Phys. Rev. A (3) 38 (1988), no. 10, 5038-5044.

[Mo] J. Moser, The analytic invariants of an areapreserving mapping near a hyperbolic fixed point. Comm. Pure Appl. Math. 9 (1956), 673-692.

[N] R. G. Newton, Scattering theory of waves and particles. Second edition. Texts and Monographs in Physics. Springer-Verlag, New York-Berlin, 1982.

[N2] R. G. Newton, Inverse Schrödinger scattering in three dimensions. Texts and Monographs in Physics. Springer-Verlag, Berlin, 1989.
[N3] R. G. Newton, Construction of potentials from the phase shifts at fixed energy. J. Mathematical Phys. 3 (1962), 75-82.

[PM] R. Pérez-Marco, Convergence or generic divergence of the Birkhoff normal form. Ann. of Math. (2) 157 (2003), no. 2, 557-574.

[PS] V. M. Petkov and L. N. Stoyanov, Geometry of Reflecting Rays and Inverse Spectral Problems, John Wiley and Sons, N.Y. (1992).

[PT] G. Popov and P. Topalov, Invariants of isospectral deformations and spectral rigidity, Comm. Partial Differential Equations 37 (2012), no. 3, 369-446.

[R] Lord J. W. S. Rayleigh, The Theory of Sound, Dover, New York (1945).

[Sab] P. C. Sabatier, General method for the inverse scattering problem at fixed energy. J. Mathematical Phys. 8 (1967), 905-918.

[Sa] M. Saprykina, Domain of analyticity of normalizing transformations. Nonlinearity 19 (2006), no. 7, 1581-1599.

[SS] B. Schmidt and C. Sutton, Two remarks on the length spectrum of a Riemannian manifold. Proc. Amer. Math. Soc. 139 (2011), no. 11, 4113-4119.

[Sch] D. Schueth, Isospectral manifolds with different local geometries. J. Reine Angew. Math. 534 (2001), 41-94.

[S] K. F. Siburg, Symplectic invariants of elliptic fixed points. Comment. Math. Helv. 75 (2000), no. 4, 681-700.

[Sj] J. Sjöstrand, Resonances associated to a closed hyperbolic trajectory in dimension 2. Asymptot. Anal. 36 (2003), no. 2, 93-113.

[St] S. Sternberg, Group theory and physics. Cambridge University Press, Cambridge, 1994.

[Su] T. Sunada, Riemannian coverings and isospectral manifolds. Ann. of Math. (2) 121 (1985), no. 1, 169-186.

[Sz] Z. Szabó, Locally non-isometric yet super isospectral spaces. Geom. Funct. Anal. 9 (1999), no. 1, $185-214$

[U] K. Uhlenbeck, Generic properties of eigenfunctions. Amer. J. Math. 98 (1976), no. 4, 1059-1078.

[Z1] S. Zelditch, The inverse spectral problem. With an appendix by Johannes Sjöstrand and Maciej Zworski. Surv. Differ. Geom., IX, Surveys in differential geometry. Vol. IX, 401-467, Int. Press, Somerville, MA, 2004

[Z2] S. Zelditch, Inverse spectral problem for analytic domains, II: Z2-symmetric domains, Ann. of Math. (2) 170 (2009), no. 1, 205-269.

[Z3] S. Zelditch, The inverse spectral problem for surfaces of revolution. J. Differential Geom. 49 (1998), no. 2, 207-264.

[Z4] S. Zelditch, Wave invariants at elliptic closed geodesics. Geom. Funct. Anal. 7 (1997), no. 1, $145-213$

[Z5] S. Zelditch, Isospectrality in the FIO category. J. Differential Geom. 35 (1992), no. 3, 689-710.

[Zu] N. T. Zung, Convergence versus integrability in Birkhoff normal form. Ann. of Math. (2) 161 (2005), no. $1,141-156$.

[Zw] M. Zworski, Semiclassical analysis. Graduate Studies in Mathematics, 138. American Mathematical Society, Providence, RI (2012), MR2952218. 